\title{
Energy and Analytic Gradients for the Orbital-Optimized Coupled-Cluster Doubles Method with the Density-Fitting Approximation: An Efficient Implementation

\author{
Uğur Bozkaya,* Aslı Ünal, and Yavuz Alagöz \\ Department of Chemistry, Hacettepe University, Ankara 06800, Turkey \\ E-mail: ugur.bozkaya@hacettepe.edu.tr
}

${ }^{*}$ To whom correspondence should be addressed 


\begin{abstract}
Efficient implementations of the orbital-optimized coupled-cluster doubles [or simply "optimized CCD", OCCD, for short] method and its analytic energy gradients with the density-fitting (DF) approach, denoted by DF-OCCD, are presented. In addition to the DF approach, the Cholesky-decomposed variant (CD-OCCD) is also implemented for energy computations. The computational cost of the DF-OCCD method is compared with that of the conventional OCCD. In the conventional OCCD, one needs to perform four-index integrals transformations at each CCD iterations, which limits its applications to large chemical systems. Our results demonstrate that DF-OCCD provides significantly lower computational costs compared to OCCD, there are almost 7-fold reductions in the computational time for the $\mathrm{C}_{5} \mathrm{H}_{12}$ molecule with the cc-pVTZ basis set. For open-shell geometries, interaction energies, and hydrogen transfer reactions, DF-OCCD provides significant improvements upon DF-CCD. Further, several factors make DF-OCCD more attractive compared to CCSD: (1) for DF-OCCD there is no need for orbital relaxation contributions in analytic gradient computations (2) active spaces can readily be incorporated into DF-OCCD (3) DF-OCCD provides accurate vibrational frequencies when symmetry-breaking problems are observed (4) in its response function, DF-OCCD avoids artificial poles; hence, excited-state molecular properties can be computed via linear response theory (5) Symmetric and asymmetric triples corrections based on DF-OCCD $[\mathrm{DF}-\mathrm{OCCD}(\mathrm{T})]$ has a significantly better performance in near degeneracy regions.
\end{abstract}




\section{Introduction}

Orbital-optimized (OO) electron correlation methods have been of significant interest in contemporary quantum chemistry. ${ }^{1-27}$ OO methods have been reported for various coupledcluster (CC) and perturbation theory methods, such as coupled-cluster doubles (CCD), ${ }^{1-3,5,13}$ coupled pair functionals, ${ }^{28,29}$ the linearized coupled-cluster doubles (LCCD), ${ }^{18,30}$ the densitycumulant functional theory (DCFT), ${ }^{21}$ the second- and third-order perturbation theory (MP2 and MP3), ${ }^{13,14,17,19,22,23,31,32}$ and the MP2.5 model. $^{24}$ Triples excitation corrections for the OO-CC methods have also been considered. ${ }^{6,15,20,33,34}$ These studies showed that the OO methods are very helpful for computations of molecular properties of challenging molecular systems, free radicals, ${ }^{10,17,18,23,35-37}$ such as symmetry-breaking problems, ${ }^{2,13,14,17}$ transition states, ${ }^{10,35-37}$ bond-breaking problems, ${ }^{15,20,38}$ weak interactions in open-shell systems, ${ }^{22,24,30,31,39}$ straightforward computation of ionization potentials ${ }^{40}$ and electron affinities, ${ }^{41}$ and evaluations of the chemical reactivity. ${ }^{42}$

One of the most common approximation for the tensor factorization of the electron repulsion integrals (ERIs) is the density fitting (DF) technique. ${ }^{22,23,30-32,43-56}$ With the help of the DF technique, one can express the four-dimensional ERIs in terms of three-dimensional tensors. In addition to to DF, the partial Cholesky decomposition (CD) of the ERI tensor is also commonly employed as a tensor decomposition approach. ${ }^{22,31,51,57-60}$ The DF and CD approximations are quite beneficial to reduce he computational time due to the reduced I/O time. In context of the OO methods, the DF and CD approaches were utilized for the OO-MP2 (OMP2 for short) energy ${ }^{10,22}$ and analytic gradients. ${ }^{23}$ Further, the DF and CD techniques were applied to the orbital-optimized MP3, MP2.5, and LCCD methods. ${ }^{30,31}$

Analytic energy gradients for electronic structure methods, which employ the DF approach, have been reported for MP2 (DF-MP2), ${ }^{52,61-64}$ the second-order coupled cluster (CC2) model, ${ }^{65,66}$ second-order multireference perturbation theory (DF-CASPT2), ${ }^{67}$ the DFOMP2, DF-OMP2.5, and DF-OMP3 methods, ${ }^{23,32}$ time-dependent local CC response theory (DF-TD-LCC2), ${ }^{68}$ the CCD and coupled-cluster singles and doubles methods (DF-CCD and 
DF-CCSD $),{ }^{54}$ and for the CCSD with perturbative triples, DF-CCSD $(T) .{ }^{55}$ However, analytic gradients with the CD approximation cannot be computed unless one employs a similar approach to that of Aquilante et al. ${ }^{69}$

In this research, energy and analytic gradients for the OCCD method ${ }^{2,13,15}$ with the DF approach is presented, which is denoted by DF-OCCD. In addition to DF, the CD approximation is also considered for the energy computations, the resulting method is denoted by CD-OCCD. The equations reported have been implemented in a new computer code, written by present authors (U.B. and A.U.), and added to the DFOCC ${ }^{22,23,30-32,52-55}$ module of the PsI4 package. ${ }^{70}$ Our new implementation has both restricted and unrestricted Hartree-Fock (RHF and UHF) versions. The DF-OCCD method is applied to bond lengths, hydrogen transfer reactions, and weak interactions.

\section{Theoretical Approach}

\subsection{Integral Tensor Decomposition Approaches}

With the help of DF and CD approximations, the atomic-orbital (AO) basis ERIs can be expressed as follows:

$$
(\mu \nu \mid \lambda \sigma)_{D F}=\sum_{Q}^{N_{\text {aux }}} b_{\mu \nu}^{Q} b_{\lambda \sigma}^{Q}
$$

In the $\mathrm{CD}$ approach, the $\mathrm{CD}$ vectors $b_{\mu \nu}^{Q}$ are obtained from the primary basis set integrals in the CD procedure, and $Q$ is a Cholesky factor. In the DF approximation, the DF factors $b_{\mu \nu}^{Q}$ may be defined as follows:

$$
b_{\mu \nu}^{Q}=\sum_{P}^{N_{\text {aux }}}(\mu \nu \mid P)\left[\mathbf{J}^{-1 / 2}\right]_{P Q},
$$


where

$$
(\mu \nu \mid P)=\iint \chi_{\mu}\left(\mathbf{r}_{\mathbf{1}}\right) \chi_{\nu}\left(\mathbf{r}_{\mathbf{1}}\right) \frac{1}{r_{12}} \varphi_{P}\left(\mathbf{r}_{\mathbf{2}}\right) d \mathbf{r}_{\mathbf{1}} d \mathbf{r}_{\mathbf{2}}
$$

and

$$
J_{P Q}=\iint \varphi_{P}\left(\mathbf{r}_{1}\right) \frac{1}{r_{12}} \varphi_{Q}\left(\mathbf{r}_{\mathbf{2}}\right) d \mathbf{r}_{\mathbf{1}} d \mathbf{r}_{\mathbf{2}}
$$

where $\chi_{\mu}(\mathbf{r})$ and $\varphi_{P}(\mathbf{r})$ are the primary and auxiliary functions, respectively. Similar to the AO basis, the molecular-orbital (MO) basis ERIs can be written as follows:

$$
(p q \mid r s)_{D F}=\sum_{Q}^{N_{a u x}} b_{p q}^{Q} b_{r s}^{Q}
$$

where $b_{p q}^{Q}$ is a $\mathrm{MO}$ basis $\mathrm{CD} / \mathrm{DF}$ tensor.

\section{$2.2 \quad$ DF-CCD Energy and Amplitude Equations}

For the orbital indexing a common notation is used: $i, j, k, l, m, n$ for occupied orbitals; $a, b, c, d, e, f$ for virtual orbitals; and $p, q, r, s, t, u, v, w$ for general spin orbitals. The correlation energy for the CCD method can be expressed as follows

$$
\Delta E=\left\langle 0\left|e^{-\hat{T}_{2}} \hat{H}_{N} e^{\hat{T}_{2}}\right| 0\right\rangle
$$

where $\hat{H}_{N}$ is the normal-ordered Hamiltonian operator, ${ }^{71,72}|0\rangle$ is the reference determinant, and $\hat{T}_{2}$ is the cluster double excitation operator:

$$
\hat{T}_{2}=\frac{1}{4} \sum_{i, j}^{o c c} \sum_{a, b}^{v i r} t_{i j}^{a b} \hat{a}^{\dagger} \hat{b}^{\dagger} \hat{j} \hat{i}
$$


where $\hat{a}^{\dagger}$ and $\hat{i}$ are the creation and annihilation operators and $t_{i j}^{a b}$ is a double excitation amplitude.

The DF-CCD correlation energy can be written explicitly as follows:

$$
\Delta E=\frac{1}{4} \sum_{i, j}^{o c c} \sum_{a, b}^{v i r} t_{i j}^{a b} \sum_{Q}^{N_{a u x}}\left(b_{i a}^{Q} b_{j b}^{Q}-b_{i b}^{Q} b_{j a}^{Q}\right)
$$

The DF-CCD amplitude equation can be written as

$$
\left\langle\Phi_{i j}^{a b}\left|e^{-\hat{T}_{2}} \hat{H}_{N} e^{\hat{T}_{2}}\right| 0\right\rangle=0
$$

where $\left\langle\Phi_{i j}^{a b}\right|$ is a doubly-excited Slater determinant. The explicit form of DF-CCD amplitude equation can be obtained from our equations for the density-fitted coupled-cluster singles and doubles (DF-CCSD) method. Hence, for details of our DF-CCD implementations, one may refer to our previous studies. ${ }^{53-55}$

\subsection{DF-CCD- $\Lambda$ Energy Functional (Lagrangian)}

It is convenient ${ }^{73,74}$ to define a Lagrangian $(\mathcal{L})$ for the DF-CCD method $(\mathrm{DF}-\mathrm{CCD}-\Lambda$ functional) as follows:

$$
\mathcal{L}=\left\langle 0\left|\left(1+\hat{\Lambda}_{2}\right) e^{-\hat{T}_{2}} \hat{H} e^{\hat{T}_{2}}\right| 0\right\rangle
$$

where $\hat{H}$ is the Hamiltonian operator and $\hat{\Lambda}_{2}$ is the CC double de-excitation operator,

$$
\hat{\Lambda}_{2}=\frac{1}{4} \sum_{i, j}^{o c c} \sum_{a, b}^{v i r} \lambda_{a b}^{i j} \hat{i}^{\dagger} \hat{j}^{\dagger} \hat{b} \hat{a}
$$

where $\lambda_{a b}^{i j}$ is a double de-excitation amplitude.

The CCD $T_{2}$-amplitude equations are obtained by differentiating that $\mathcal{L}$ with respect to $\Lambda_{2}$-amplitudes, whereas the differentiating with respect to $T_{2}$-amplitudes yields to the 
$\Lambda_{2}$-amplitude equations ${ }^{75-79}$

$$
\left\langle 0\left|\left(1+\hat{\Lambda}_{2}\right)\left[e^{-\hat{T}_{2}} \hat{H} e^{\hat{T}_{2}}-E\right]\right| \Phi_{i j}^{a b}\right\rangle=0
$$

where $E$ is the CCD total energy. The explicit form of DF-CCD $\Lambda_{2}$ amplitude equations can be obtained from our equations for the DF-CCSD method in previous studies. ${ }^{53-55}$

\subsection{The Parametrization of the DF-OCCD Wave Function}

We follow our previous formulations ${ }^{13-15,17-19,24,30,31,39,40}$ for the DF-OCCD wave function. The MO transformations may be achieved with the help of a unitary operator ${ }^{80-83}$

$$
\begin{aligned}
\tilde{\hat{p}}^{\dagger} & =e^{\hat{K}} \hat{p}^{\dagger} e^{-\hat{K}}, \\
\tilde{\hat{p}} & =e^{\hat{K}} \hat{p} e^{-\hat{K}}, \\
|\tilde{p}\rangle & =e^{\hat{K}}|p\rangle,
\end{aligned}
$$

where $e^{\hat{K}}$ is the MO rotation operator, $\tilde{\hat{p}}^{\dagger}, \tilde{\hat{p}}$, and $|\tilde{p}\rangle$ are the transformed creation, annihilation operators and a transformed spin-orbital, respectively, and $\hat{K}$ is:

$$
\hat{K}=\sum_{p, q} K_{p q} \hat{p}^{\dagger} \hat{q}=\sum_{p>q} \kappa_{p q}\left(\hat{p}^{\dagger} \hat{q}-\hat{q}^{\dagger} \hat{p}\right)
$$

hence,

$$
\mathbf{K}=\operatorname{Skew}(\boldsymbol{\kappa}),
$$

where $\left\{\kappa_{p q}\right\}$ are the MO rotation parameters. The transformed MO coefficients matrix can be written as

$$
\mathbf{C}(\boldsymbol{\kappa})=\mathbf{C}^{(\mathbf{0})} e^{\mathbf{K}}
$$


where $\mathbf{C}^{(\mathbf{0})}$ and $\mathbf{C}(\boldsymbol{\kappa})$ are the old and new MO coefficient matrices, respectively.

For DF-OCCD, the following Lagrangian can be written, ${ }^{13}$

$$
\mathcal{L}(\boldsymbol{\kappa})=\left\langle 0\left|\left(1+\hat{\Lambda}_{2}\right) e^{-\hat{T}_{2}} \hat{H}^{\kappa} e^{\hat{T}_{2}}\right| 0\right\rangle
$$

where,

$$
\hat{H}^{\kappa}=e^{-\hat{K}} \hat{H} e^{\hat{K}}
$$

Derivatives of $\mathcal{L}(\boldsymbol{\kappa})$ with respect to $\boldsymbol{\kappa}$ can be expressed as follows,

$$
\begin{gathered}
w_{p q}=\left.\frac{\partial \mathcal{L}}{\partial \kappa_{p q}}\right|_{\kappa=0}, \\
A_{p q, r s}=\left.\frac{\partial^{2} \mathcal{L}}{\partial \kappa_{p q} \partial \kappa_{r s}}\right|_{\kappa=0} .
\end{gathered}
$$

Then, a second-order series expansion can be written for $\mathcal{L}(\boldsymbol{\kappa})$ as follows:

$$
\mathcal{L}^{(2)}(\boldsymbol{\kappa})=\mathcal{L}^{(0)}+\boldsymbol{\kappa}^{\dagger} \boldsymbol{w}+\frac{1}{2} \boldsymbol{\kappa}^{\dagger} \boldsymbol{A} \boldsymbol{\kappa}
$$

where $\boldsymbol{\kappa}$ is the MO rotation vector, $\boldsymbol{w}$ is the MO gradient vector, and $\boldsymbol{A}$ is the orbital Hessian matrix. Hence, one can obtain the following equation by minimizing $\mathcal{L}$ with respect to $\boldsymbol{\kappa}$,

$$
\kappa=-\boldsymbol{A}^{-1} \boldsymbol{w}
$$




\subsection{Response Density Matrices}

Particle density matrices (PDMs) are central for the evaluation of the energy derivatives. The CCD one-particle density matrix (OPDM) can be defined as follows: ${ }^{13,15}$

$$
\gamma_{p q}=\frac{1}{2} P_{+}(p q)\left\langle 0\left|\left(1+\hat{\Lambda}_{2}\right) e^{-\hat{T}_{2}} \hat{p}^{\dagger} \hat{q} e^{\hat{T}_{2}}\right| 0\right\rangle,
$$

The OPDM can be partitioned into the reference and correlation parts as follows:

$$
\gamma_{p q}=\gamma_{p q}^{r e f}+\gamma_{p q}^{c o r r}
$$

where $\gamma_{p q}^{r e f}$ and $\gamma_{p q}^{c o r r}$ are the reference and correlation parts of OPDM, respectively.

With the DF/CD approximation we can avoid the formation of four-index two-particle density matrix (TPDM), we may form a three-index TPDM, instead. The three-index TPDM is defined as follows: ${ }^{22,23,52,54}$

$$
\Gamma_{p q}^{Q}=\frac{1}{2} \hat{P}_{+}(p q) \sum_{r, s}\left\langle 0\left|\left(1+\hat{\Lambda}_{2}\right) e^{-\hat{T}_{2}} \hat{p}^{\dagger} \hat{r}^{\dagger} \hat{s} \hat{q} e^{\hat{T}_{2}}\right| 0\right\rangle b_{r s}^{Q}
$$

where $\hat{P}_{+}(p q)$ is the symmetrizer, which has the following effect on a tensor:

$$
\hat{P}_{+}(p q) A_{p q}=A_{p q}+A_{q p}
$$

The three-index TPDM can be decomposed as in the case of OPDM as follows: ${ }^{22}$

$$
\Gamma_{p q}^{Q}=\Gamma_{p q}^{Q(\text { ref })}+\Gamma_{p q}^{Q(\text { corr })}+\Gamma_{p q}^{Q(s e p)}
$$

where $\Gamma_{p q}^{Q(\text { ref })}$ and $\Gamma_{p q}^{Q(c o r r)}$ are the reference and correlation parts of TPDM, respectively, and $\Gamma_{p q}^{Q(s e p)}$ is the separable part of TPDM. The explicit form of DF-CCD response PDMs can be obtained from our equations for the DF-CCSD method in previous studies. ${ }^{54,55}$ 
Then, the energy of the DF-CCD- $\Lambda$ functional may be re-written in terms of PDMs as follows

$$
\mathcal{L}=\sum_{p, q} \gamma_{p q} h_{p q}+\frac{1}{2} \sum_{Q}^{N_{a u x}} \sum_{p, q} \Gamma_{p q}^{Q} b_{p q}^{Q}
$$

\subsection{Orbital Gradient}

The MO gradient is defined as follows: ${ }^{13,14,18,24}$

$$
w_{p q}=2\left(F_{p q}-F_{q p}\right)
$$

where $F_{p q}$ is the generalized-Fock matrix (GFM). Similar to the TPDM, the GFM can be partitioned into reference, correlation, and separable components as follows: ${ }^{22,23,52}$

$$
F_{p q}=F_{p q}^{r e f}+F_{p q}^{c o r r}+F_{p q}^{s e p}
$$

For $F_{p q}^{r e f}$ and $F_{p q}^{s e p}$ the DF-REF basis integrals, while for $F_{p q}^{c o r r}$ the DF-CC basis integrals are employed. The explicit form of the GFM is reported in our previous studies. ${ }^{22,23,52}$

\subsection{The Orbital Optimization Procedure}

The DF-OCCD wave function is described by the set of $\boldsymbol{\kappa}, \boldsymbol{t}_{\mathbf{2}}$, and $\boldsymbol{\lambda}_{\mathbf{2}}$ parameters. Similar to previous studies, ${ }^{2,13,14,17-19}$ the $\boldsymbol{t}_{\mathbf{2}}, \boldsymbol{\lambda}_{\mathbf{2}}$, and $\boldsymbol{\kappa}$ parameters are simultaneously optimized. The parameters $\boldsymbol{\kappa}$ are obtained from Eq.(24) using the approximate Hessian introduced in

our 2014 study. ${ }^{22}$ The direct inversion in the iterative subspace (DIIS) method ${ }^{84}$ is utilized to accelerate the convergence. ${ }^{18}$ 


\subsection{DF-OCCD Analytic Gradients}

The DF-OCCD energy is minimized with respect to the MO parameters. Hence, there is no need to consider orbital relaxation effects for analytic gradients. However, the DF-OCCD energy is not stationary with respect to CC amplitudes; hence, one need to consider their response in the gradient expression. Hence, we employ the Lagrangian of Eq.(10). The first derivative of the energy may be written as follows ${ }^{73,74,83,85-90}$

$$
\left.\frac{\mathrm{d} E}{\mathrm{~d} x}\right|_{x=x_{0}}=\left.\frac{\partial \mathcal{L}}{\partial x}\right|_{x=x_{0}}
$$

The first derivative equation can be cast into the following form: ${ }^{23}$

$$
\frac{\mathrm{d} E}{\mathrm{~d} x}=\sum_{p, q} \gamma_{p q} h_{p q}^{x}-\sum_{p, q} F_{p q} S_{p q}^{x}+\sum_{Q}^{N_{a u x}} \sum_{p q} \widetilde{\Gamma}_{p q}^{Q}(Q \mid p q)^{x}-\sum_{P, Q}^{N_{\text {aux }}} \Gamma_{P Q} \mathbf{J}_{P Q}^{x}
$$

Two- and three-index TPDMs are defined by ${ }^{23,52}$

$$
\begin{aligned}
\widetilde{\Gamma}_{p q}^{Q} & =\sum_{P}^{N_{\text {aux }}} \Gamma_{p q}^{P}\left[\mathbf{J}^{-1 / 2}\right]_{P Q}, \\
\Gamma_{P Q} & =\frac{1}{2} \sum_{p, q} c_{p q}^{P} \widetilde{\Gamma}_{p q}^{Q}=\frac{1}{2} \sum_{p, q} \widetilde{\Gamma}_{p q}^{P} c_{p q}^{Q}, \\
c_{p q}^{Q} & =\sum_{P}^{N_{\text {aux }}} b_{p q}^{P}\left[\mathbf{J}^{-1 / 2}\right]_{P Q} .
\end{aligned}
$$

Then, the PDMs and GFM are back-transformed into the AO basis. ${ }^{91-93}$

$$
\begin{aligned}
F_{\mu \nu} & =\sum_{p q} C_{\mu p} C_{\nu q} F_{p q}, \\
\gamma_{\mu \nu} & =\sum_{p q} C_{\mu p} C_{\nu q} \gamma_{p q}, \\
\Gamma_{\mu \nu}^{Q} & =\sum_{p q} C_{\mu p} C_{\nu q} \widetilde{\Gamma}_{p q}^{Q},
\end{aligned}
$$

where $F_{\mu \nu}, \gamma_{\mu \nu}$, and $\Gamma_{\mu \nu}^{Q}$ are the AO basis GFM, OPDM, and three-index TPDM, respectively. 
The two-index TPDM may be expressed as follows:

$$
\begin{aligned}
\Gamma_{P Q} & =\frac{1}{2} \sum_{p, q} c_{p q}^{P} \widetilde{\Gamma}_{p q}^{Q}=\frac{1}{2} \sum_{\mu \nu} c_{\mu \nu}^{P} \Gamma_{\mu \nu}^{Q}, \\
c_{\mu \nu}^{Q} & =\sum_{P}^{N_{a u x}} b_{\mu \nu}^{P}\left[\mathbf{J}^{-1 / 2}\right]_{P Q},
\end{aligned}
$$

Hence, the final analytic gradient expression in the AO basis can be written as follows ${ }^{52}$

$$
\frac{\mathrm{d} E}{\mathrm{~d} x}=\sum_{\mu \nu} \gamma_{\mu \nu} h_{\mu \nu}^{x}-\sum_{\mu \nu} F_{\mu \nu} S_{\mu \nu}^{x}+\sum_{Q}^{N_{\text {aux }}} \sum_{\mu \nu} \Gamma_{\mu \nu}^{Q}(Q \mid \mu \nu)^{x}-\sum_{P, Q}^{N_{\text {aux }}} \Gamma_{P Q} \mathbf{J}_{P Q}^{x} .
$$

\section{Results and Discussion}

The efficiency of the OCCD ${ }^{2,13}$ and DF-OLCCD methods were compared using a set of alkanes. For the alkanes set, Dunning's correlation-consistent polarized valence triple- $\zeta$ basis set (cc-pVTZ) was employed. ${ }^{94,95}$ The cc-pVTZ-JKFIT ${ }^{48}$ and cc-pVTZ-RI ${ }^{96}$ auxiliary basis sets were employed for the reference and correlation energies, respectively, as the fitting basis sets for cc-pVTZ. Further, the MP2, DF-CCD, DF-OCCD, CCSD, and CCSD(T) methods were applied to a set of molecules ${ }^{18}$ for comparison of equilibrium geometries. For geometries, Dunning's correlation-consistent polarized core and valence quadruple- $\zeta$ (cc-pCVQZ) basis set was used. ${ }^{94,95}$ The cc-pVQZ-JKFIT ${ }^{48}$ and cc-pVQZ-RI ${ }^{96}$ auxiliary basis sets were used for reference and correlation energies, respectively, as fitting basis sets for cc-pCVQZ. Geometry optimizations were performed with analytic gradients for each method. Moreover, hydrogen transfer reaction energies (HTRE) ${ }^{17,24,97}$ were regarded to investigate the performance of DFOCCD. For the HTRE set, the cc-pVTZ primary basis set, and its canonical auxiliary basis sets (cc-pVTZ-JKFIT and cc-pVTZ-RI ${ }^{96}$ ) were employed. In the CD-OCCD computations, a CD threshold of $10^{-4}$ was employed.

Further, noncovalent interaction complexes (the A24 and O20 sets) ${ }^{39,98}$ were considered to investigate the performance of DF-OCCD for open-shell noncovalent interactions. For weak 
interactions, single-point energies were obtained at optimized geometries, and the total energies were extrapolated to complete basis set (CBS) limits. ${ }^{99,100}$ The two-point extrapolation approach of Halkier et al. ${ }^{101}$ was used for this purpose. ${ }^{102}$ In the two-point extrapolation procedure, for the A24 set the aug-cc-pVDZ and aug-cc-pVTZ basis sets were employed, while for the $\mathrm{O} 20$ set the aug-cc-pVTZ and aug-cc-pVQZ basis sets were used. For the noncovalent interaction complexes, the corresponding auxiliary basis sets, aug-cc-pVXZ-JKFIT ${ }^{48}$ and aug-cc-pVXZ-RI, ${ }^{96}$ were employed as fitting basis sets. For the He atom, the aug-cc-pVXZJKFIT basis set is not available; hence, we employed the def2-QZVPP-JKFIT auxiliary basis set. Similarly, for the Li atom, the def2-QZVPP-JKFIT and def2-QZVPP-RI basis sets were used. Counterpoise corrections are considered for all intermolecular interaction energies. ${ }^{103}$

\subsection{The Efficiency of DF-OCCD}

A set of alkanes is considered to investigate the efficiency of the OCCD and DF-OLCCD methods. The computational time for the OCCD and DF-OCCD methods are presented graphically in Figure 1. The DF-OCCD method dramatically reduces the computational cost compared to the conventional OCCD, there are 6.8-fold reductions in the computational time compared to OCCD for the largest member $\left(\mathrm{C}_{5} \mathrm{H}_{12}\right)$ of the alkanes set. The dramatic difference between the computational cost of DF-OLCCD and OLCCD is mainly arising from the efficiency of DF integral transformation procedure due to reduced $\mathrm{I} / \mathrm{O}$ time. The

accuracy of the DF approximation is well assessed in previous studies, ${ }^{22,30-32,54,55}$ it introduces quite negligible errors compared to the conventional methods: noncovalent energies exhibit mean absolute errors (MAEs) of 0.01-0.09 $\mathrm{kcal} \mathrm{mol}^{-1}$, equilibrium bond lengths has a MAE value of $10^{-4} \AA$, and vibrational frequencies yield a MAE of $0.5 \mathrm{~cm}^{-1} \cdot{ }^{22,30-32,54,55}$

\subsection{Geometries}

We start with a set of closed-shell as the first step of our investigation. ${ }^{24}$ Table S1 of the supporting information reports bond lengths of molecules considered. Errors in bond lengths 
for the MP2, DF-CCD, DF-OCCD, CCSD, and CCSD(T) methods with respect to experiment are presented in Figure 2, while the MAE values are illustrated in Figure 3. The MAE values are 0.006 (MP2), 0.009 (DF-CCD), 0.007 (DF-OCCD), 0.007 (CCSD), and 0.002 $[\operatorname{CCSD}(\mathrm{T})] \AA$. The DF-CCD method yields the largest error compared to the experiment, while $\operatorname{CCSD}(\mathrm{T})$ yields the lowest error as expected. The DF-OCCD, OCCD, and CCSD methods yield the same MAE value. The DF-OCCD method remarkably enhances the MP2 and DF-CCD results, by $25 \%$ and $31 \%$, respectively.

Next, we study a set of open-shell molecules. ${ }^{104}$ Table S2 of the supporting information reports bond lengths of open-shell molecules considered. Errors in bond lengths for the MP2, DF-CCD, DF-OCCD, CCSD, and CCSD(T) methods with respect to experiment are presented in Figure 4, while the MAE values are illustrated in Figure 5. The MAE values are 0.014 (MP2), 0.015 (DF-CCD), 0.013 (DF-OCCD), 0.012 (CCSD), and 0.007 [CCSD(T)] $\AA$. The DF-CCD method again yields the largest error compared with the experiment, while $\operatorname{CCSD}(\mathrm{T})$ yields the lowest error as expected. Further, the performances of DF-OCCD and CCSD are almost identical.

\subsection{Hydrogen Transfer Reactions}

It was demonstrated that the canonical methods, such as MP2, MP3, and LCCD, dramatically fail for the HTREs, which include free radicals. ${ }^{17,18,24,97}$ It was reported that the OO methods, such as OMP2 and OLCCD, provide remarkably better performance than their canonical versions (MP2 and LCCD), providing 5- and 6-fold lower MAEs compared with

MP2 and OO linearized CCD (OLCCD). ${ }^{17,18,24}$ Hence, we consider the same test set ${ }^{17}$ to assess the performance of DF-OCCD.

Table 1 reports the HTRE values (in kcal mol-1) from the MP2, DF-CCD, DF-OCCD, CD-OCCD, CCSD, and CCSD(T) methods at the CBS limit. Errors with respect to $\operatorname{CCSD}(\mathrm{T})$ are presented in Figure 6, whereas the MAE values (Figure 7) are 14.6 (MP2), 8.2 (DF-CCD), 0.5 (DF-OCCD), 0.5 (CD-OCCD), and 0.5 (CCSD) kcal mol ${ }^{-1}$. Hence, the 
results of DF-OCCD, CD-OCCD, and CCSD are identical and significantly better than those of MP2 and DF-CCD. Furthermore, our results indicate that there is a reduction in DF-CCD errors by more than a factor of 16 when optimized orbitals are used, and comparing to MP2 there is a more than 29-fold decrease in errors.

\subsection{Noncovalent Interactions}

In this section we consider weak interactions to investigate the performance of DF-OCCD, and we start with the A24 set. ${ }^{98}$ For the A24 set, interaction energies from the MP2, DFCCD, DF-OCCD, CD-OCCD, and CCSD methods at the CBS limit are reported in Table 2. Errors and the mae values with respect to reference energies (Table 2) are depicted in Figure 8 and Figure 9, respectively. The MAE values are 0.11 (MP2), 0.25 (DF-CCD), 0.25 (DF-OCCD), 0.26 (CD-OCCD), and 0.26 (CCSD) kcal mol${ }^{-1}$. Hence, the performance of DF-CCD, DF-OCCD, CD-OCCD, and CCSD are identical. It is well-known that the HF orbitals are reliably used in most of the closed-shell systems. It is unexpected that the DF-OCCD and CCSD methods yield a larger MAE value compared with MP2. But, it is consistent with a previous study. ${ }^{105}$

Finally, we consider the $\mathrm{O} 20$ set $^{22,39}$ to investigate the performance of DF-OCCD. Table 3 reports noncovalent interaction energies (in $\mathrm{kcal} \mathrm{mol}^{-1}$ ) for the $\mathrm{O} 20$ set from the MP2, DF-CCD, DF-OCCD, CCSD, and CCSD(T) methods at the CBS limit. Errors with respect to $\operatorname{CCSD}(\mathrm{T})$ are presented in Figure 10, whereas the MAE values are illustrated in Figure 11. The MAE values are 0.60 (MP2), 0.50 (DF-CCD), 0.36 (DF-OCCD), 0.38 (CD-OCCD), and 0.34 (CCSD) kcal mol${ }^{-1}$. Hence, there is a noticeable improvement upon DF-CCD when optimized orbitals are used. Further, the performance of DF-OCCD, CD-OCCD, and CCSD are virtually the same. 


\section{Conclusions}

The density-fitted orbital-optimized CCD (DF-OCCD) and its analytic energy gradients have been presented. In addition to DF-OCCD, the Cholesky decomposed variant (CD-OCCD) is also presented for energy computations. Results from the DF-OCCD method have been obtained for closed- and open-shell molecular geometries, HTREs, and interaction energies of noncovalent complexes for comparison with those from the MP2, DF-CCD, CCSD, and $\operatorname{CCSD}(\mathrm{T})$ methods.

For the minimization of the MOs of the DF-OCCD wave function, a Lagrangian-based technique has been utilized as in the case of previous OO methods. ${ }^{13,14,18,22,24,30,31}$ Both the OCCD and CCSD methods scale formally as $O\left(N^{6}\right)$, where $N$ is the number of basis functions. However, the main drawback of the conventional OCCD is that one needs to perform four-index integral transformations at each CC iteration. ${ }^{2,13}$ Even though, the MO transformation scales as $O\left(N^{5}\right)$, it can not be performed in the core memory, except for the very small molecular systems; hence, it is the most expensive part of CC iterations due to the slow I/O procedure. However, with the DF approach, the cost of MO transformations is reduced to $O\left(N^{4}\right)$, and memory requirements are significantly reduced, by a factor of $N$. Therefore, with the DF approach, it is possible to perform MO transformations in the core memory in many cases. Even if there is no enough memory for the DF algorithm, it is still significantly faster in out-of-core algorithm. Hence, the DF approximation dramatically reduce the cost of the conventional OCCD method.

For molecular geometries, HTREs, and interaction energies the DF-OCCD significantly improves upon MP2 and DF-CCD. For example, for hydrogen transfer reactions there are 16- and 29-fold reductions in errors when DF-OCCD is employed compared with DF-CCD and MP2, respectively. Furthermore, for these test systems, the performance of DF-OCCD and CCSD are virtually the same. However, several factors make DF-OCCD more attractive compared to CCSD: (1) for DF-OCCD there is no need for orbital relaxation contributions in analytic gradient computations (2) active spaces ${ }^{5}$ can readily be incorporated into OCCD (3) 
DF-OCCD provides accurate vibrational frequencies when symmetry-breaking problems ${ }^{2,13}$ are observed (4) in its response function, DF-OCCD avoids artificial poles; hence, excitedstate molecular properties can be computed via linear response theory ${ }^{4,7,106}$ (5) Symmetric and asymmetric triples corrections based on DF-OCCD $[\mathrm{OCCD}(\mathrm{T})]$ has a significantly better performance in near degeneracy regions. ${ }^{15}$

\section{Supporting Information Available}

(1) Computed and experimental bond lengths for the closed- and open-shell molecules.

This material is available free of charge via the Internet at http://pubs.acs.org/.

\section{Acknowledgement}

This research was supported by the Scientific and Technological Research Council of Turkey (TÜBİTAK-118Z916).

\section{References}

(1) Scuseria, G. E.; Schaefer, H. F. The Optimization of Molecular Orbitals for Coupled Cluster Wavefunctions. Chem. Phys. Lett. 1987, 142, 354-358.

(2) Sherrill, C. D.; Krylov, A. I.; Byrd, E. F. C.; Head-Gordon, M. Energies and Analytic Gradients for a Coupled-Cluster Doubles Model Using Variational Brueckner Orbitals Application to Symmetry Breaking in $\mathrm{O}_{4}^{+}$. J. Chem. Phys. 1998, 109, 4171-4181.

(3) Krylov, A. I.; Sherrill, C. D.; Byrd, E. F. C.; Head-Gordon, M. Size-Consistent Wave Functions for Nondynamical Correlation Energy: The Valence Active Space Optimized Orbital Coupled-Cluster Doubles Model. J. Chem. Phys. 1998, 109, 10669-10678.

(4) Pedersen, T. B.; Koch, H.; Hättig, C. Gauge Invariant Coupled Cluster Response Theory. J. Chem. Phys. 1999, 110, 8318-8327. 
(5) Krylov, A. I.; Sherrill, C. D.; Head-Gordon, M. Excited States Theory for Optimized Orbitals and Valence Optimized Orbitals Coupled-Cluster Doubles Models. J. Chem. Phys. 2000, 113, 6509-6527.

(6) Gwaltney, S. R.; Sherrill, C. D.; Head-Gordon, M.; Krylov, A. I. Second Order Perturbation Corrections to Singles and Doubles Coupled-Cluster Methods: General Theory and Applications to the Valence Optimized Doubles Model. J. Chem. Phys. 2000, $113,3548-3560$.

(7) Pedersen, T. B.; Fernández, B.; Koch, H. Gauge Invariant Coupled Cluster Response Theory Using Optimized Nonorthogonal Orbitals. J. Chem. Phys. 2001, 114, 69836992.

(8) Köhn, A.; Olsen, J. Orbital-Optimized Coupled-Cluster Theory Does not Reproduce the Full Configuration-Interaction Limit. J. Chem. Phys. 2005, 122, 084116.

(9) Lochan, R. C.; Head-Gordon, M. Orbital-Optimized Opposite-Spin Scaled SecondOrder Correlation: An Economical Method to Improve the Description of Open-Shell Molecules. J. Chem. Phys. 2007, 126, 164101.

(10) Neese, F.; Schwabe, T.; Kossmann, S.; Schirmer, B.; Grimme, S. Assessment of Orbital-Optimized, Spin-Component Scaled Second-Order Many-Body Perturbation Theory for Thermochemistry and Kinetics. J. Chem. Theory Comput. 2009, 5, 30603073 .

(11) Kurlancheek, W.; Head-Gordon, M. Violations of N-representability from SpinUnrestricted Orbitals in Møller-Plesset perturbation Theory and Related DoubleHybrid Density Functional Theory. Mol. Phys. 2009, 107, 1223-1232.

(12) Kossmann, S.; Neese, F. Correlated Ab Initio Spin Densities for Larger Molecules: Orbital-Optimized Spin-Component-Scaled MP2 Method. J. Phys. Chem. A 2010, 114, 11768. 
(13) Bozkaya, U.; Turney, J. M.; Yamaguchi, Y.; Schaefer, H. F.; Sherrill, C. D. Quadratically Convergent Algorithm for Orbital Optimization in the Orbital-Optimized Coupled-Cluster Doubles Method and in Orbital-Optimized Second-Order MøllerPlesset Perturbation Theory. J. Chem. Phys. 2011, 135, 104103.

(14) Bozkaya, U. Orbital-Optimized Third-Order Møller-Plesset Perturbation Theory and Its Spin-Component and Spin-Opposite Scaled Variants: Application to Symmetry Breaking Problems. J. Chem. Phys. 2011, 135, 224103.

(15) Bozkaya, U.; Schaefer, H. F. Symmetric and Asymmetric Triple Excitation Corrections for the Orbital-Optimized Coupled-Cluster Doubles Method: Improving Upon $\operatorname{CCSD}(\mathrm{T})$ and $\operatorname{CCSD}(\mathrm{T})$ ?: Preliminary Application. J. Chem. Phys. 2012, 136, 204114 .

(16) Kurlancheek, W.; Lawler, K.; Lochan, R. C.; Head-Gordon, M. Exploring the Competition Cetween Localization and Delocalization of the Neutral Soliton Defect in Polyenyl Chains With the Orbital Optimized Second Order Opposite Spin Method. J. Chem. Phys. 2012, 136, 054113.

(17) Bozkaya, U.; Sherrill, C. D. Analytic Energy Gradients for the Orbital-Optimized Second-Order Møller-Plesset Perturbation Theory. J. Chem. Phys. 2013, 138, 184103.

(18) Bozkaya, U.; Sherrill, C. D. Orbital-Optimized Coupled-Electron Pair Theory and Its Analytic Gradients: Accurate Equilibrium Geometries, Harmonic Vibrational Frequencies, and Hydrogen Transfer Reactions. J. Chem. Phys. 2013, 139, 054104.

(19) Bozkaya, U. Analytic Energy Gradients for the Orbital-Optimized Third-Order MøllerPlesset Perturbation Theory. J. Chem. Phys. 2013, 139, 104116.

(20) Robinson, J. B.; Knowles, P. J. Rigorously Extensive Orbital-Invariant Renormalized Perturbative Triples Corrections From Quasi-Variational Coupled Cluster Theory. J. Chem. Phys. 2013, 138, 074104. 
(21) Sokolov, A. Y.; Schaefer, H. F. Orbital-Optimized Density Cumulant Functional Theory. J. Chem. Phys. 2013, 139, 204110.

(22) Bozkaya, U. Orbital-Optimized Second-Order Perturbation Theory with DensityFitting and Cholesky Decomposition Approximations: An Efficient Implementation. J. Chem. Theory Comput. 2014, 10, 2371-2378.

(23) Bozkaya, U. Analytic Energy Gradients and Spin Multiplicities for Orbital-Optimized Second-Order Perturbation Theory with Density-Fitting Approximation: An Efficient Implementation. J. Chem. Theory Comput. 2014, 10, 4389-4399.

(24) Bozkaya, U.; Sherrill, C. D. Orbital-Optimized MP2.5 and Its Analytic Gradients: Approaching CCSD(T) Quality for Noncovalent Interactions. J. Chem. Phys. 2014, $141,204105$.

(25) Lee, J.; Head-Gordon, M. Regularized Orbital-Optimized Second-Order Møller-Plesset Perturbation Theory: A Reliable Fifth-Order-Scaling Electron Correlation Model with Orbital Energy Dependent Regularizers. J. Chem. Theory Comput. 2018, 14, 5203-5219.

(26) Lee, J.; Head-Gordon, M. Two single-reference approaches to singlet biradicaloid problems: Complex, restricted orbitals and approximate spin-projection combined with regularized orbital-optimized Møller-Plesset perturbation theory. J. Chem. Phys. 2019, 150, 244106.

(27) Bertels, L. W.; Lee, J.; Head-Gordon, M. Third-Order Møller-Plesset Perturbation Theory Made Useful? Choice of Orbitals and Scaling Greatly Improves Accuracy for Thermochemistry, Kinetics, and Intermolecular Interactions. J. Phys. Chem. Lett. 2019, 10, 4170-4176.

(28) Kollmar, C.; Heßelmann, A. The Role of Orbital Transformations in Coupled-Pair Functionals. Theor. Chem. Acc. 2010, 127, 311-325. 
(29) Kollmar, C.; Neese, F. An Orbital-Invariant and Strictly Size Extensive Post-HartreeFock Correlation Functional. J. Chem. Phys. 2011, 135, 084102.

(30) Bozkaya, U. Orbital-Optimized Linearized Coupled-Cluster Doubles with DensityFitting and Cholesky Decomposition Approximations: An Efficient Implementation. Phys. Chem. Chem. Phys. 2016, 18, 11362-11373.

(31) Bozkaya, U. Orbital-Optimized MP3 and MP2.5 with Density-Fitting and Cholesky Decomposition Approximations. J. Chem. Theory Comput. 2016, 12, 1179-1188.

(32) Bozkaya, U. Analytic Energy Gradients for Orbital-Optimized MP3 and MP2.5 with the Density-Fitting Approximation: An Efficient Implementation. J. Comp. Chem. 2018, 39, 351-360.

(33) Robinson, J. B.; Knowles, P. J. Approximate Variational Coupled Cluster Theory. J. Chem. Phys. 2011, 135, 044113.

(34) Robinson, J. B.; Knowles, P. J. Quasi-Variational Coupled Cluster Theory. J. Chem. Phys. 2012, 136, 054114.

(35) Soydaş, E.; Bozkaya, U. Assessment of Orbital-Optimized Third-Order Møller-Plesset Perturbation Theory and Its Spin-Component and Spin-Opposite Scaled Variants for Thermochemistry and Kinetics. J. Chem. Theory Comput. 2013, 9, 1452-1460.

(36) Soydaş, E.; Bozkaya, U. Assessment of the Orbital-Optimized Coupled-Electron Pair Theory for Thermochemistry and Kinetics: Improving on CCSD and CEPA(1). J. Comp. Chem. 2014, 35, 1073-1081.

(37) Soydaş, E.; Bozkaya, U. Assessment of Orbital-Optimized Third-Order Møller-Plesset Perturbation Theory and Its Spin-Component and Spin-Opposite Scaled Variants for Thermochemistry and Kinetics. J. Chem. Theory Comput. 2015, 11, 1564-1573. 
(38) Robinson, J. B.; Knowles, P. J. Benchmark Quasi-Variational Coupled Cluster Calculations of Multiple Bond Breaking. J. Chem. Theory Comput. 2012, 8, 2653-2660.

(39) Soydaş, E.; Bozkaya, U. Accurate Open-Shell Noncovalent Interaction Energies from the Orbital-Optimized Møller-Plesset Perturbation Theory: Achieving CCSD Quality at the MP2 Level by Orbital Optimization. J. Chem. Theory Comput. 2013, 9, 46794683.

(40) Bozkaya, U. The Extended Koopmans' Theorem for Orbital-Optimized Methods: Accurate Computation of Ionization Potentials. J. Chem. Phys. 2013, 139, 154105.

(41) Bozkaya, U. Accurate Electron Affinities from the Extended Koopmans Theorem Based on Orbital-Optimized Methods. J. Chem. Theory Comput. 2014, 10, 20412048.

(42) Yildiz, D.; Bozkaya, U. Assessment of the Extended Koopmans' Theorem for the Chemical Reactivity: Accurate Computations of Chemical Potentials, Chemical Hardnesses, and Electrophilicity Indices. J. Comp. Chem. 2016, 37, 345-353.

(43) Whitten, J. L. Coulombic Potential Energy Integrals and Approximations. J. Chem. Phys. 1973, 58, 4496-4501.

(44) Dunlap, B. I.; Connolly, J. W. D.; Sabin, J. R. On Some Approximations in Applications of X? Theory. J. Chem. Phys. 1979, 71, 3396-3402.

(45) Feyereisen, M.; Fitzgerald, G.; Komornicki, A. Use of Approximate Integrals in Ab Initio Theory. An Application in MP2 Energy Calculations. Chem. Phys. Lett. 1993, 208, 359-363.

(46) Vahtras, O.; Almlöf, J.; Feyereisen, M. W. Integral Approximations for LCAO-SCF Calculations. Chem. Phys. Lett. 1993, 213, 514-518. 
(47) Rendell, A. P.; Lee, T. J. Coupled-cluster Theory Employing Approximate Integrals: An Approach to Avoid the Input/Output and Storage Bottlenecks. J. Chem. Phys. 1994, 101, 400-408.

(48) Weigend, F. A Fully Direct RI-HF Algorithm: Implementation, Optimised Auxiliary Basis Sets, Demonstration of Accuracy and Efficiency. Phys. Chem. Chem. Phys. 2002, 4, 4285-4291.

(49) Sodt, A.; Subotnik, J. E.; Head-Gordon, M. Linear Scaling Density Fitting. J. Chem. Phys. 2006, 125, 194109.

(50) Werner, H.-J.; Schütz, M. An Efficient Local Coupled Cluster Method for Accurate Thermochemistry of Large Systems. J. Chem. Phys. 2011, 135, 144116.

(51) DePrince, A. E.; Sherrill, C. D. Accuracy and Efficiency of Coupled-Cluster Theory Using Density Fitting/Cholesky Decomposition, Frozen Natural Orbitals, and a t1Transformed Hamiltonian. J. Chem. Theory Comput. 2013, 9, 2687-2696.

(52) Bozkaya, U. Derivation of General Analytic Gradient Expressions for Density-Fitted Post-Hartree-Fock Methods: An Efficient Implementation for the Density-Fitted Second-Order Møller-Plesset Perturbation Theory. J. Chem. Phys. 2014, 141, 124108.

(53) Bozkaya, U. A Noniterative Asymmetric Triple Excitation Correction for The DensityFitted Coupled-Cluster Singles and Doubles Method: Preliminary Applications. J. Chem. Phys. 2016, 144, 144108.

(54) Bozkaya, U.; Sherrill, C. D. Analytic Energy Gradients for the Coupled-Cluster Singles and Doubles Method with the Density-Fitting Approximation. J. Chem. Phys. 2016, $144,174103$.

(55) Bozkaya, U.; Sherrill, C. D. Analytic Energy Gradients for The Coupled-Cluster Sin- 
gles and Doubles with Perturbative Triples Method with the Density-Fitting Approximation. J. Chem. Phys. 2017, 147, 044104-044114.

(56) Bozkaya, U. Efficient Implementation of the Second-Order Quasidegenerate Perturbation Theory with Density-Fitting and Cholesky Decomposition Approximations: Is It Possible To Use Hartree-Fock Orbitals for a Multiconfigurational Perturbation Theory? J. Chem. Theory Comput. 2019, 15, 4415-4429.

(57) Beebe, N. H. F.; Linderberg, J. Simplifications in the Generation and Transformation of Two-Electron Integrals in Molecular Calculations. Int. J. Quant. Chem. 1977, 12, 683-705.

(58) Roeggen, I.; Wisloff-Nilssen, E. On the Beebe-Linderberg Two-Electron Integral Approximation. Chem. Phys. Lett. 1986, 132, 154-160.

(59) Koch, H.; de Meras, A. S.; Pedersen, T. B. Reduced Scaling in Electronic Structure Calculations Using Cholesky Decompositions. J. Chem. Phys. 2003, 118, 9481-9484.

(60) Aquilante, F.; Pedersen, T. B.; Lindh, R. Low-Cost Evaluation of the Exchange Fock Matrix From Cholesky and Density Fitting Representations of the Electron Repulsion Integrals. J. Chem. Phys. 2007, 126, 194106.

(61) Weigend, F.; Häser, M. RI-MP2: First Derivatives and Global Consistency. Theor. Chem. Acc. 1997, 97, 331-340.

(62) Hättig, C.; Hellweg, A.; Köhn, A. Distributed Memory Parallel-Implementation of Energies and Gradients for Second-Order Møller-Plesset Perturbation Theory With the Resolution-of-the-Identity Approximation. Phys. Chem. Chem. Phys. 2006, 8, 1159-1169.

(63) Distasio, R. A.; Steele, R. P.; Rhee, Y. M.; Shao, Y.; Head-Gordon, M. An Improved Algorithm for Analytical Gradient Evaluation in Resolution-of-the-Identity Second- 
Order Møller-Plesset Perturbation Theory: Application to Alanine Tetrapeptide Conformational Analysis. J. Comp. Chem. 2007, 28, 839-856.

(64) Schütz, M.; Werner, H.-J.; Lindh, R.; Manby, F. R. Analytical Energy Gradients for Local Second-Order Møller-Plesset Perturbation Theory Using Density Fitting Approximations. J. Chem. Phys. 2004, 121, 737.

(65) Hättig, C. Geometry Optimizations With the Coupled-Cluster Model CC2 Using the Resolution-of-the-Identity Approximation. J. Chem. Phys. 2003, 118, 7751.

(66) Köhn, A.; Hättig, C. Analytic Gradients for Excited States in the Coupled-Cluster Model CC2 Employing the Resolution-of-the-Identity Approximation. J. Chem. Phys. 2003, 119, 5021.

(67) Györffy, W.; Shiozaki, T.; Knizia, G.; Werner, H.-J. Analytical Energy Gradients for Second-Order Multireference Perturbation Theory Using Density Fitting. J. Chem. Phys. 2013, 138, 104104.

(68) Ledermüller, L.; Schütz, M. Local CC2 Response Method Based on the Laplace Transform: Analytic Energy Gradients for Ground and Excited States. J. Chem. Phys. 2014, 140, 164113.

(69) Aquilante, F.; Lindh, R.; Pedersen, T. B. Analytic Derivatives for the Cholesky Representation of the Two-Electron Integrals. J. Chem. Phys. 2008, 129, 034106.

(70) Smith, D. G. A.; Burns, L. A.; Simmonett, A. C.; Parrish, R. M.; Schieber, M. C.; Galvelis, R.; Kraus, P.; Kruse, H.; Remigio, R. D.; Alenaizan, A.; James, A. M.; Lehtola, S.; Misiewicz, J. P.; Scheurer, M.; Shaw, R. A.; Schriber, J. B.; Xie, Y.; Glick, Z. L.; Sirianni, D. A.; O’Brien, J. S.; Waldrop, J. M.; Kumar, A.; Hohenstein, E. G.; Pritchard, B. P.; Brooks, B. R.; Schaefer, H. F.; Sokolov, A. Y.; Patkowski, K.; DePrince, A. E.; Bozkaya, U.; King, R. A.; Evangelista, F. A.; Tur- 
ney, J. M.; Crawford, T. D.; Sherrill, C. D. Psi4 1.4: Open-source Software for HighThroughput Quantum Chemistry. J. Chem. Phys. 2020, 152, 184108.

(71) Shavitt, I.; Bartlett, R. J. Many-Body Methods in Chemistry and Physics, 1st ed.; Cambridge Press: New York, 2009; pp 443-449.

(72) Crawford, T. D.; Schaefer, H. F. An Introduction to Coupled Cluster Theory for Computational Chemists. Rev. Comp. Chem. 2000, 14, 33-136.

(73) Helgaker, T.; Jørgensen, P. Analytical Calculation of Geometrical Derivatives in Molecular Electronic Structure Theory. Adv. Quantum Chem. 1988, 19, 183.

(74) Jørgensen, P.; Helgaker, T. Møller-Plesset Energy Derivatives. J. Chem. Phys. 1988, $89,1560$.

(75) Salter, E. A.; Trucks, G. W.; Bartlett, R. J. Analytic Energy Derivatives in Many-Body Methods. I. First Derivatives. J. Chem. Phys. 1989, 90, 1752.

(76) Gauss, J.; Stanton, J. F.; Bartlett, R. J. Coupled-Cluster Open-Shell Analytic Gradients: Implementation of the Direct Pproduct Decomposition Approach in Energy Gradient Calculations. J. Chem. Phys. 1991, 95, 2623-2638.

(77) Gauss, J.; Stanton, J. F.; Bartlett, R. J. Analytic Evaluation of Energy Gradients at the Coupled-Cluster Singles and Doubles Level Using Quasi-Restricted Hartree-Fock Open-Shell Reference Functions. J. Chem. Phys. 1991, 95, 2639-2645.

(78) Gauss, J.; Stanton, J. F. Coupled-Cluster Calculations of Nuclear Magnetic Resonance Chemical Shifts. J. Chem. Phys. 1995, 103, 3561.

(79) Gauss, J.; Stanton, J. F. Analytic Gradients for the Coupled-Cluster Singles, Doubles, and Triples (CCSDT) Model. J. Chem. Phys. 2001, 116, 1773.

(80) Dalgaard, E.; Jørgensen, P. Optimization of Orbitals for Multiconfigurational Reference States. J. Chem. Phys. 1978, 69, 3833-3844. 
(81) Helgaker, T.; Jørgensen, P.; Olsen, J. Molecular Electronic Structure Theory, 1st ed.; John Wiley \& Sons: New York, 2000; pp 496-504.

(82) Shepard, R. The Multiconfiguration Self-Consistent Field Method. Adv. Chem. Phys. 1987, 69, 63-200.

(83) Shepard, R. In Modern Electronic Structure Theory Part I, 1st ed.; Yarkony, D. R., Ed.; Advanced Series in Physical Chemistry Vol.2; World Scientific Publishing Company: London, 1995; pp 345-458.

(84) Pulay, P. Convergence Acceleration of Iterative Sequences. The Case of SCF Iteration. Chem. Phys. Lett. 1980, 73, 393.

(85) Helgaker, T.; Jørgensen, P.; Handy, N. A numerically Stable Procedure for Calculating Møller-Plesset Energy Derivatives, Derived Using the Theory of Lagrangians. Theor. Chem. Acc. 1989, 76, 227-245.

(86) Helgaker, T.; Jørgensen, P. Configuration-Interaction Energy Derivatives in a Fully Variational Formulation. Theor. Chem. Acc. 1989, 75, 111-127.

(87) Helgaker, T. U.; Almlöf, J. A second-Quantization Approach to the Analytical Evaluation of Response Properties for Perturbation-Dependent Basis Sets. Int. J. Quant. Chem. 1984, 26, 275-291.

(88) Helgaker, T. U. In Geometrical Derivatives of Energy Surfaces and Molecular Properties; Jørgensen, P., Simons, J., Eds.; Springer: Reidel, Dordrecht, 1986; pp 1-16.

(89) Simons, J.; Helgaker, T. U.; Jørgensen, P. Higher Molecular-Deformation Derivatives of the Configuration-Interaction Energy. Chem. Phys. 1984, 86, 413-432.

(90) Helgaker, T. In The Encyclopedia of Computational Chemistry; Schleyer, P. R., Allinger, N. L., Clark, T., Gasteiger, J., Kollman, P. A., Schaefer, H. F., Schreiner, P. R., Eds.; Wiley: Chichester, 1998; pp 1157-1169. 
(91) Rice, J. E.; Amos, R. D. On the Efficient Evaluation of Analytic Energy Gradients. Chem. Phys. Lett. 1985, 122, 585-590.

(92) Yamaguchi, Y.; Osamura, Y.; Goddard, J. D.; Schaefer, H. F. A New Dimension to Quantum Chemistry: Analytic Derivative Methods in Ab Initio Molecular Electronic Structure Theory; Oxford University Press: New York, 1994; pp 29-52, 128-143.

(93) Yamaguchi, Y.; Schaefer, H. F. In Handbook of High-Resolution Spectroscopies; Quack, M., Merkt, F., Eds.; John Wiley \& Sons, 2011; pp 325-362.

(94) Dunning, T. H. Gaussian Basis Sets for Use in Correlated Molecular Calculations. I. The Atoms Boron Through Neon and Hydrogen. J. Chem. Phys. 1989, 90, 1007-1023.

(95) Woon, D. E.; Dunning, T. H. Gaussian Basis Sets for Use in Correlated Molecular Calculations. V. Core-Valence Basis Sets for Boron through Neon. J. Chem. Phys. 1995, 103, 4572-4585.

(96) Weigend, F.; Köhn, A.; Hättig, C. Efficient Use of the Correlation Consistent Basis Sets in Resolution of the Identity MP2 Calculations. J. Chem. Phys. 2002, 116, 31753183.

(97) Temelso, B.; Sherrill, C. D.; Merkle, R. C.; Freitas, R. A. High-Level Ab Initio Studies of Hydrogen Abstraction from Prototype Hydrocarbon Systems. J. Phys. Chem. A 2006, 110, 11160-11173.

(98) Rezác, J.; Hobza, P. Describing Noncovalent Interactions beyond the Common Approximations: How Accurate Is the "Gold Standard, " CCSD(T) at the Complete Basis Set Limit? J. Chem. Theory Comput. 2013, 9, 2151-2155.

(99) Feller, D. The Use of Systematic Sequences of Wave Functions for Estimating the Complete Basis Set, Full Configuration Interaction Limit in Water. J. Chem. Phys. 1993, 98, 7059-7071. 
(100) Helgaker, T.; Klopper, W.; Koch, H.; Noga, J. Basis-Set Convergence of Correlated Calculations on Water. J. Chem. Phys. 1997, 106, 9639-9646.

(101) Halkier, A.; Helgaker, T.; Jørgensen, P.; Klopper, W.; Olsen, J. Basis-set Convergence of the Energy in Molecular Hartree-Fock Calculations. Chem. Phys. Lett. 1999, 302, $437-446$.

(102) Bozkaya, U.; Soydaş, E.; Filiz, B. State-of-the-art Computations of Dipole Moments Using Analytic Gradients of High-Level Density-Fitted Coupled-Cluster Methods with Focal-Point Approximations. J. Comp. Chem. 2020, 41, 769-779.

(103) Boys, S. F.; Bernardi, F. The Calculation of Small Molecular Interactions by the Differences of Separate Total Energies. Some Procedures With Reduced Errors. Mol. Phys. 1970, 19, 553-566.

(104) Byrd, E. F. C.; Sherrill, C. D.; Head-Gordon, M. The Theoretical Prediction of Molecular Radical Species: a Systematic Study of Equilibrium Geometries and Harmonic Vibrational Frequencies. J. Phys. Chem. A 2001, 105, 9736.

(105) Copan, A. V.; Sokolov, A. Y.; Schaefer, H. F. Benchmark Study of Density Cumulant Functional Theory: Thermochemistry and Kinetics. J. Chem. Theory Comput. 2014, 10, 2389-2398.

(106) McAlexander, H. R.; Mach, T. J.; Crawford, T. D. Localized Optimized Orbitals, Coupled Cluster Theory, and Chiroptical Response Properties. Phys. Chem. Chem. Phys. 2012, 14, 7830-7836.

(107) Dhont, G. S. F.; van Lenthe, J. H.; Groenenboom, G. C.; van der Avoird, A. Ab Initio Calculation of the $\mathrm{NH}\left({ }^{3} \Sigma^{-}\right)-\mathrm{NH}\left({ }^{3} \Sigma^{-}\right)$Interaction Potentials in the Quintet, Triplet, and Singlet States. J. Chem. Phys. 2005, 123, 184302. 


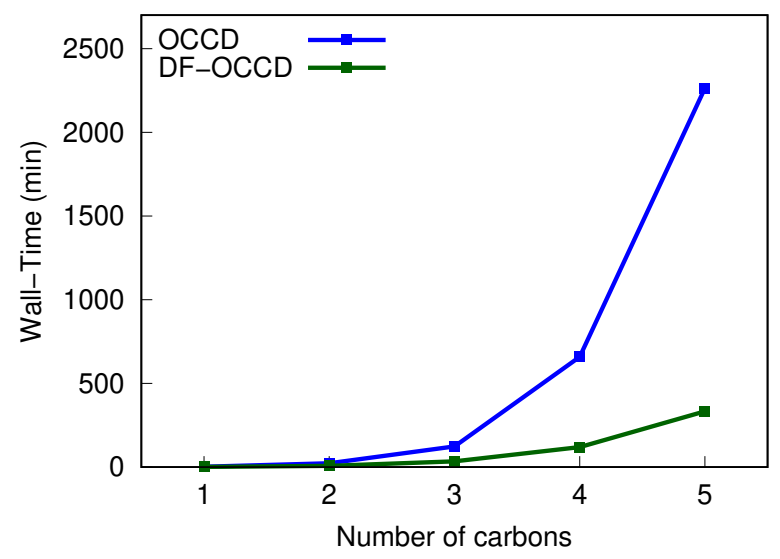

Figure 1: Wall-time (in min) for computations of single-point energies for the $\mathrm{C}_{n} \mathrm{H}_{2 n+2}(\mathrm{n}=1-$ 5 ) set from the OCCD and DF-OCCD methods with the cc-pVTZ basis set. All computations were performed with a $10^{-7}$ energy convergence tolerance on a single node (1 cores) Intel(R) Xeon(R) Gold 5218 CPU @ 2.30 GHz computer (memory 500 GB). 


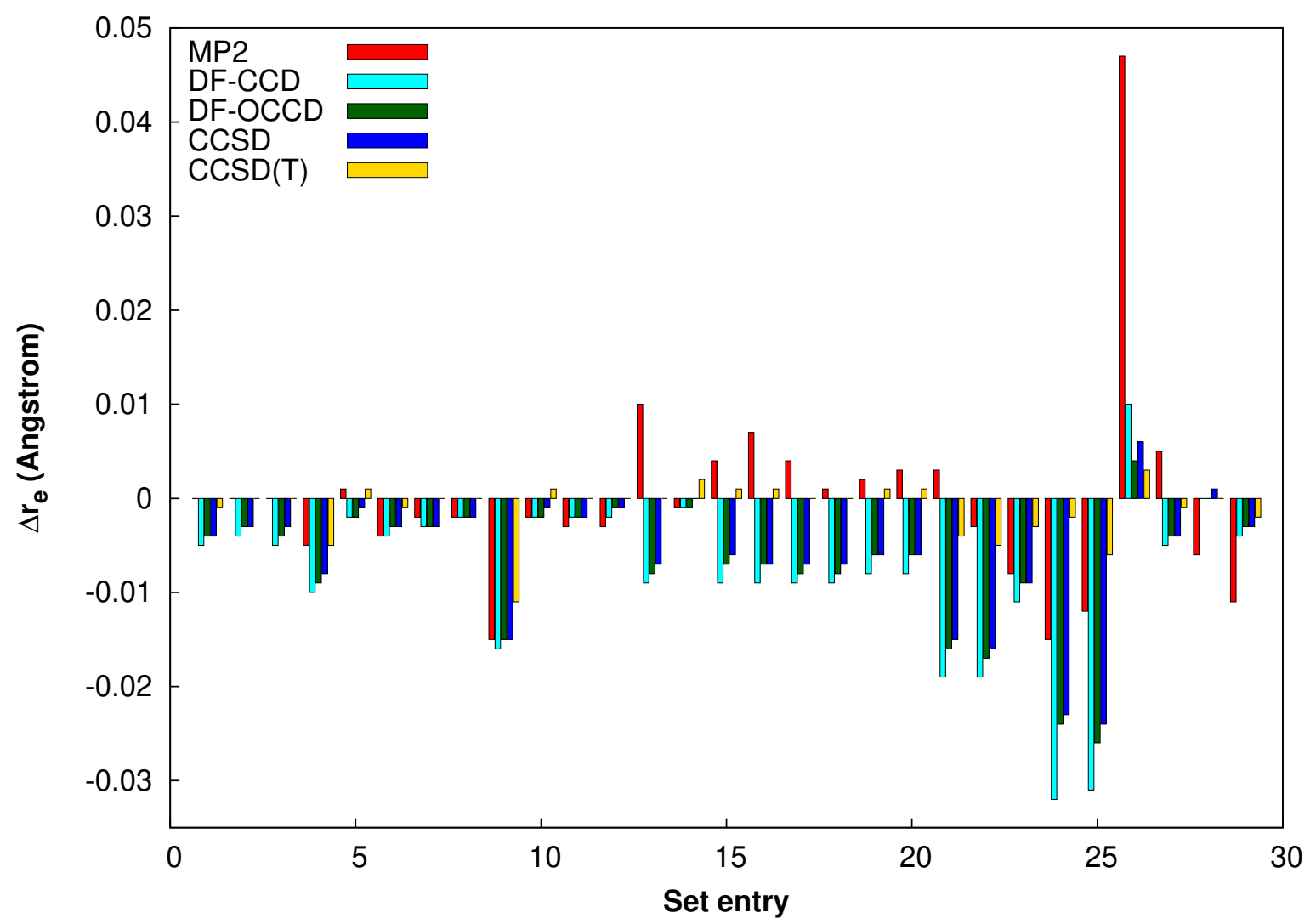

Figure 2: Errors in bond lengths of closed-shell molecules for the MP2, DF-CCD, DFOCCD, CCSD, and CCSD $(\mathrm{T})$ methods with respect to experiment (the cc-pCVQZ basis set was employed). 


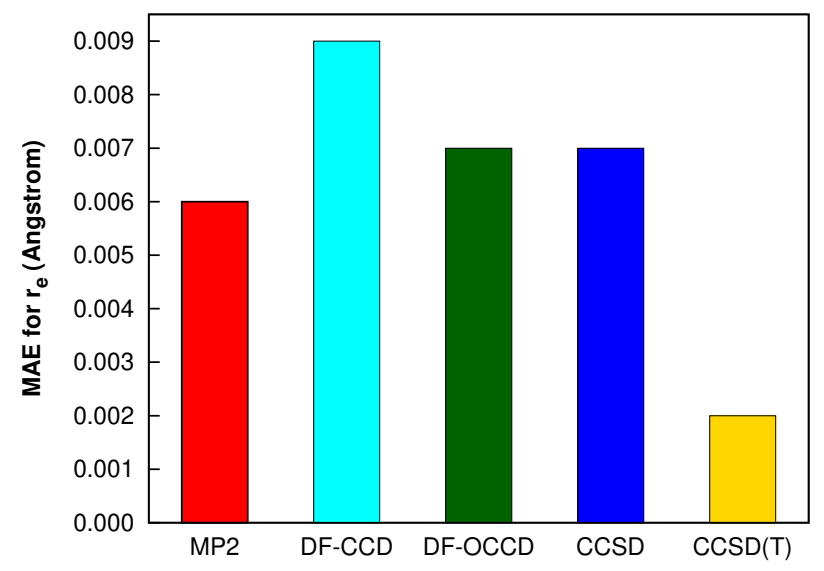

Figure 3: Mean absolute errors in bond lengths of closed-shell molecules for the MP2, DFCCD, DF-OCCD, CCSD, and CCSD(T) methods with respect to experiment (the cc-pCVQZ basis set was employed). 


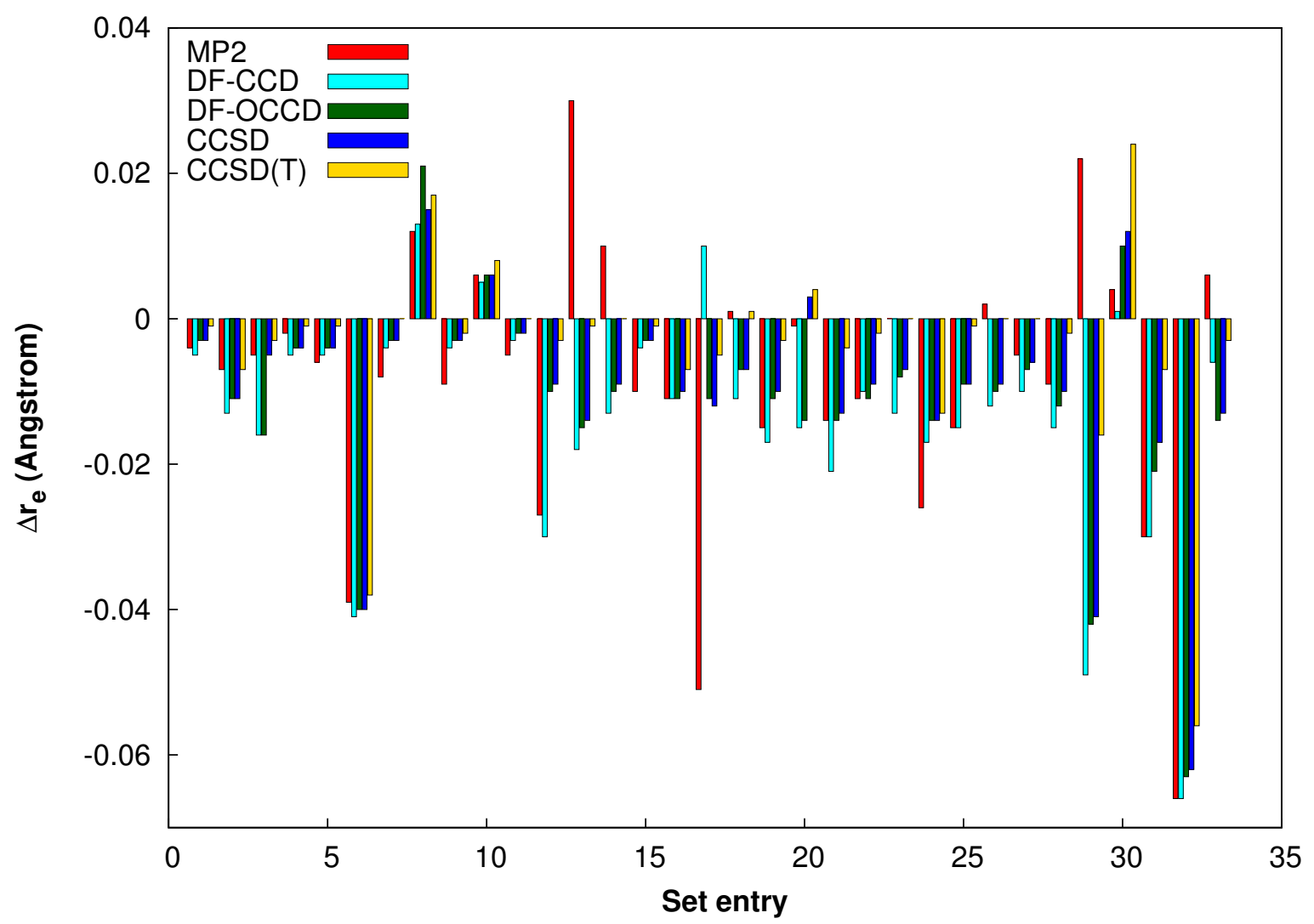

Figure 4: Errors in bond lengths of open-shell molecules from Byrd et al. ${ }^{104}$ for the MP2, DF-CCD, DF-OCCD, CCSD, and CCSD(T) methods with respect to experiment (the ccpCVQZ basis set was employed). 


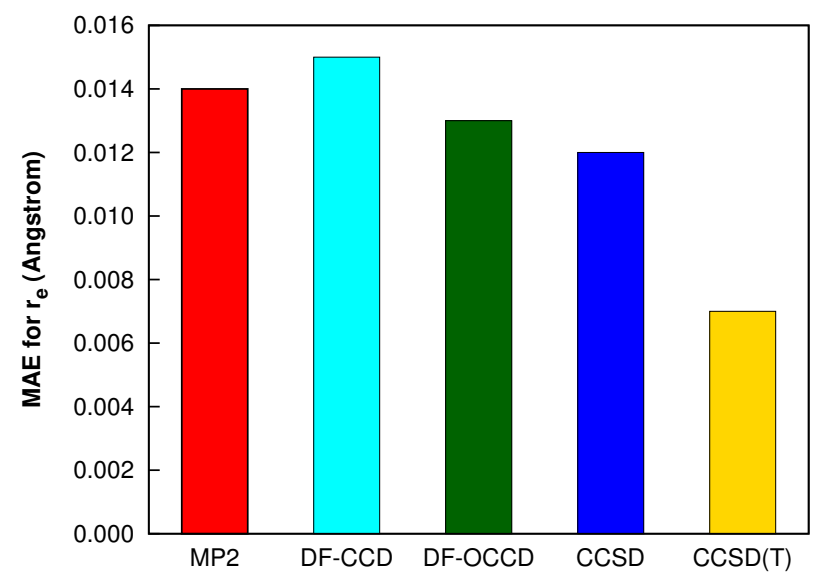

Figure 5: Mean absolute errors in bond lengths of open-shell molecules from Byrd et al. ${ }^{104}$ for the MP2, DF-CCD, DF-OCCD, CCSD, and CCSD(T) methods with respect to experiment (the cc-pCVQZ basis set was employed). 


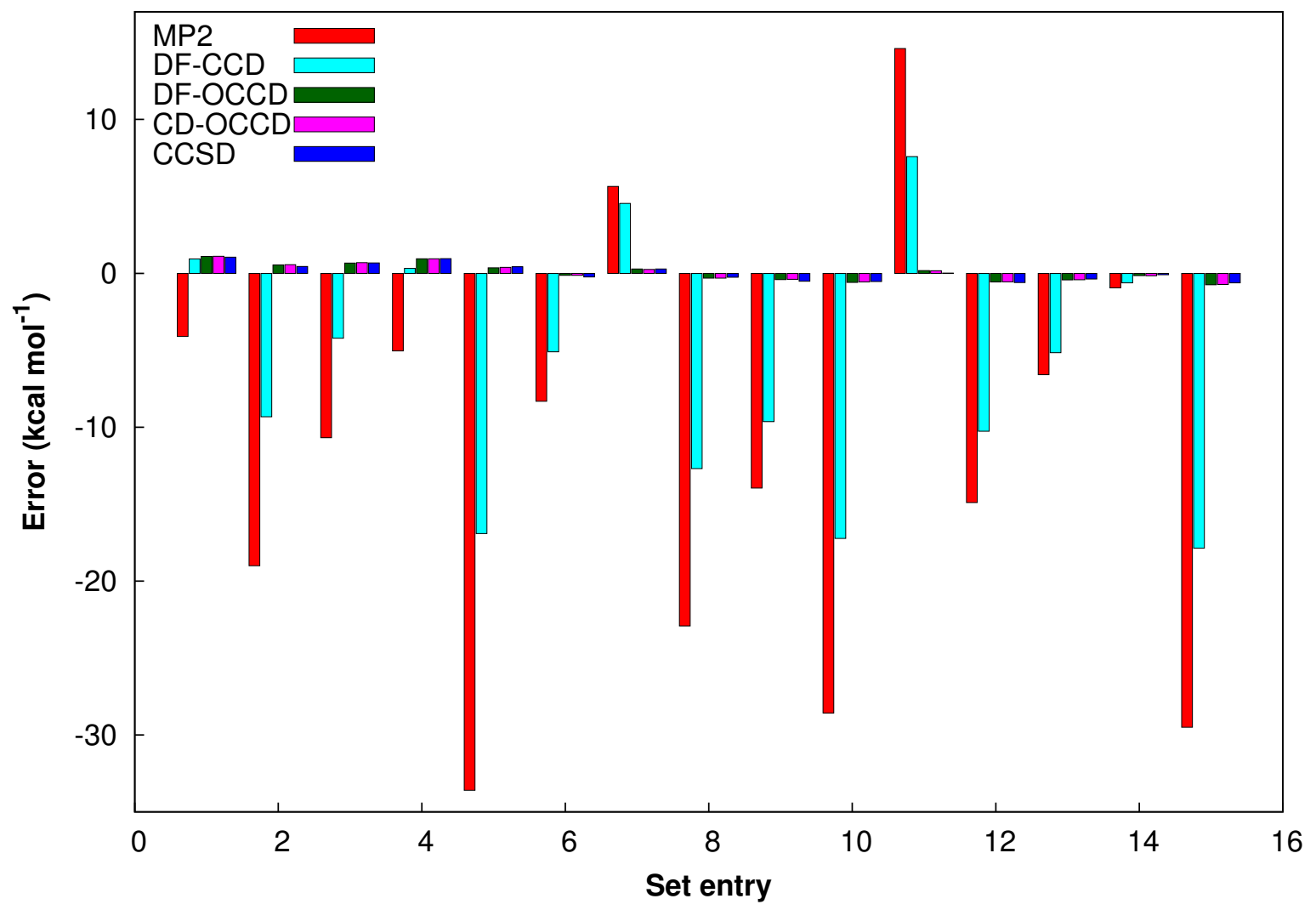

Figure 6: Errors in hydrogen transfer reaction energies (Table 1) for the MP2, DF-CCD, DF-OCCD, CD-OCCD, and CCSD methods with respect to $\operatorname{CCSD}(\mathrm{T})$, all in the CBS limit. 


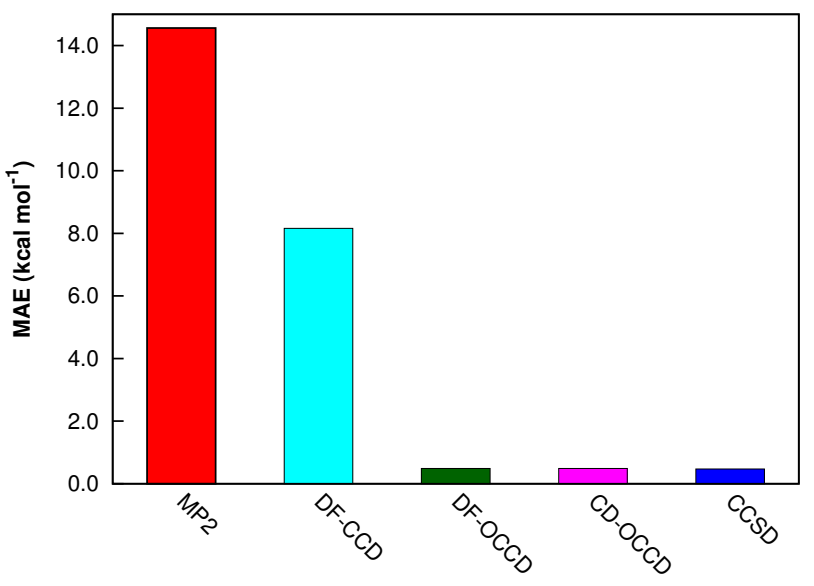

Figure 7: Mean absolute errors in hydrogen transfer reaction energies (Table 1) for the MP2, DF-CCD, DF-OCCD, CD-OCCD, OCCD, and CCSD methods with respect to CCSD $(\mathrm{T})$, all in the CBS limit. 


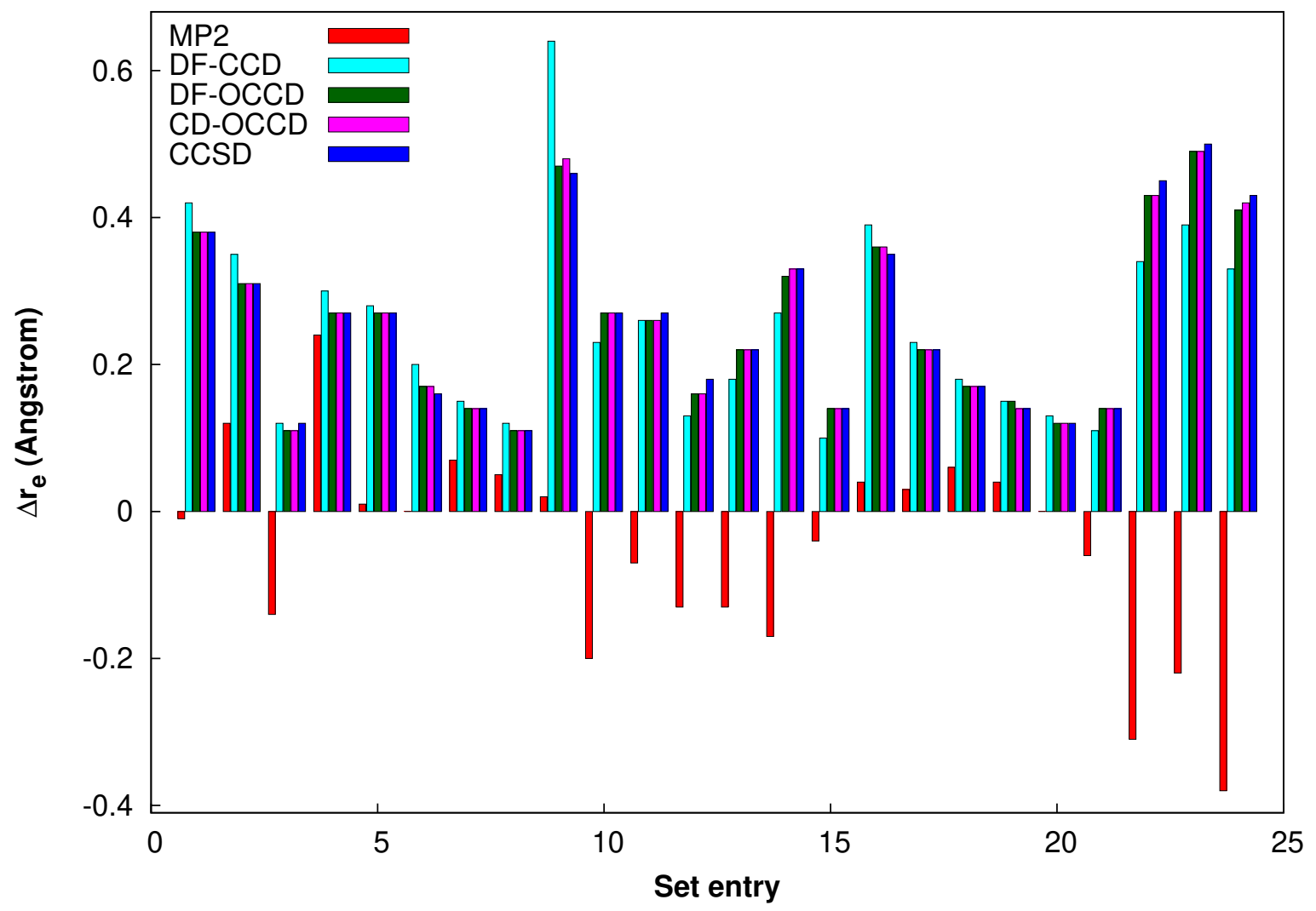

Figure 8: Errors in closed-shell noncovalent interaction energies (Table 2) for the MP2, DFCCD, DF-OCCD, CD-OCCD, and CCSD methods (all in the CBS limit) with respect to reference energies. ${ }^{98}$ 


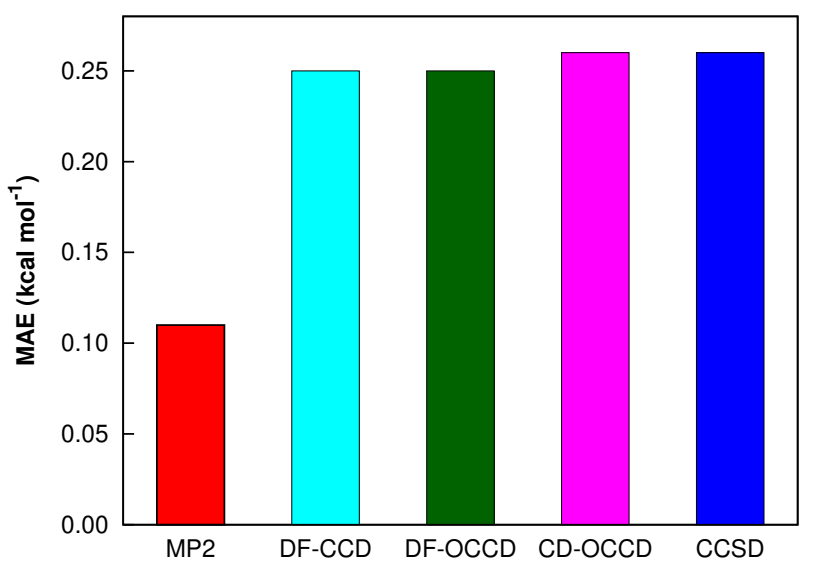

Figure 9: Mean absolute errors in closed-shell noncovalent interaction energies (Table 2) for the MP2, DF-CCD, DF-OCCD, CD-OCCD, and CCSD methods (all in the CBS limit) with respect to reference energies. ${ }^{98}$ 


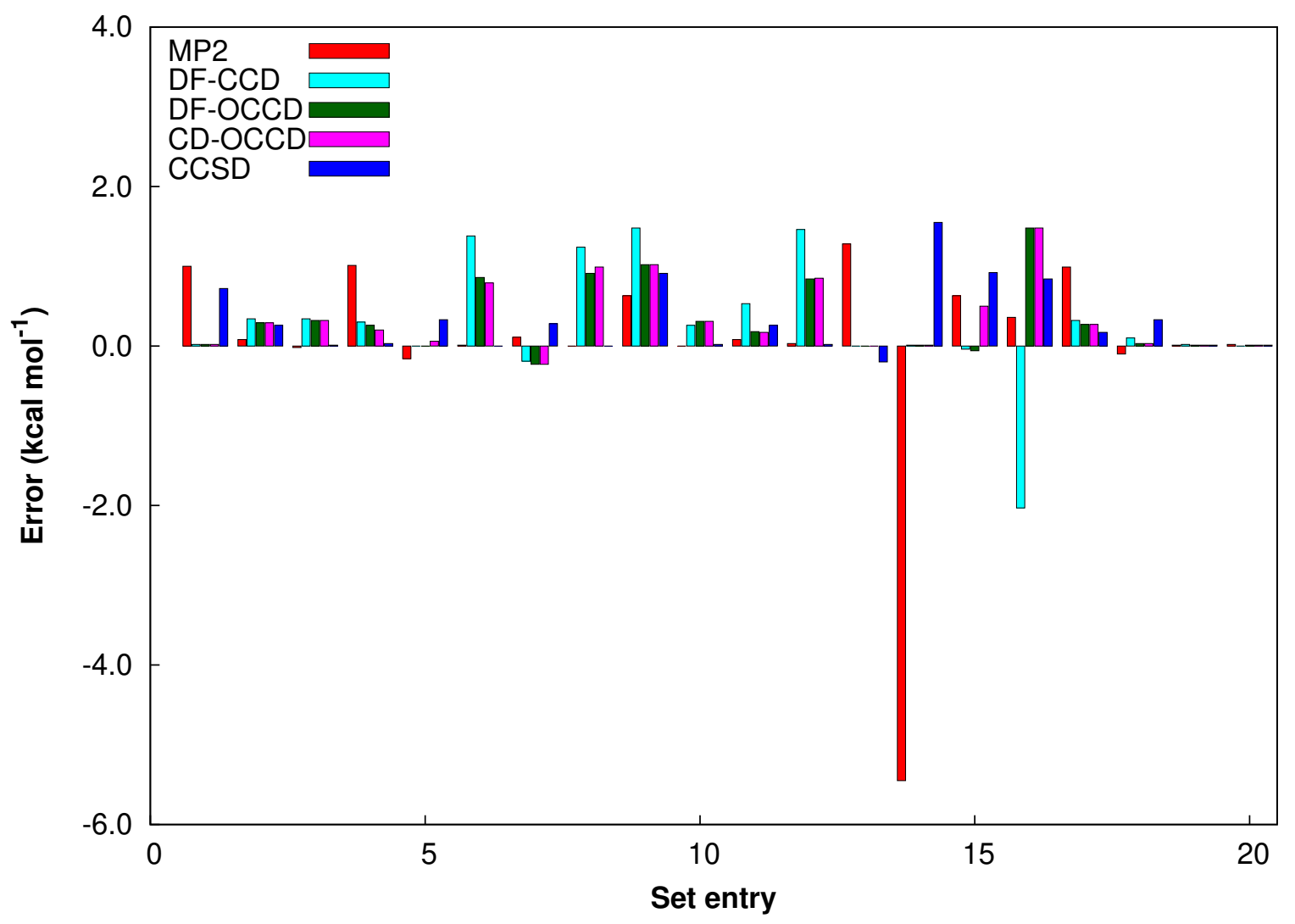

Figure 10: Errors in open-shell noncovalent interaction energies (Table 2) for the MP2, DFCCD, DF-OCCD, CD-OCCD, and CCSD methods (all in the CBS limit) with respect to reference energies. ${ }^{98}$ 


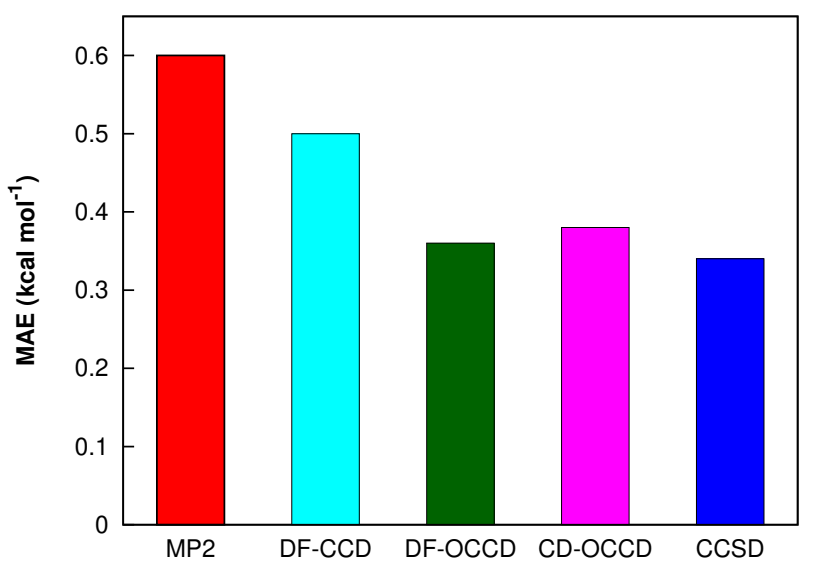

Figure 11: Mean absolute errors in open-shell noncovalent interaction energies (Table 2) for the MP2, DF-CCD, DF-OCCD, CD-OCCD, and CCSD methods (all in the CBS limit) with respect to reference energies. ${ }^{98}$ 


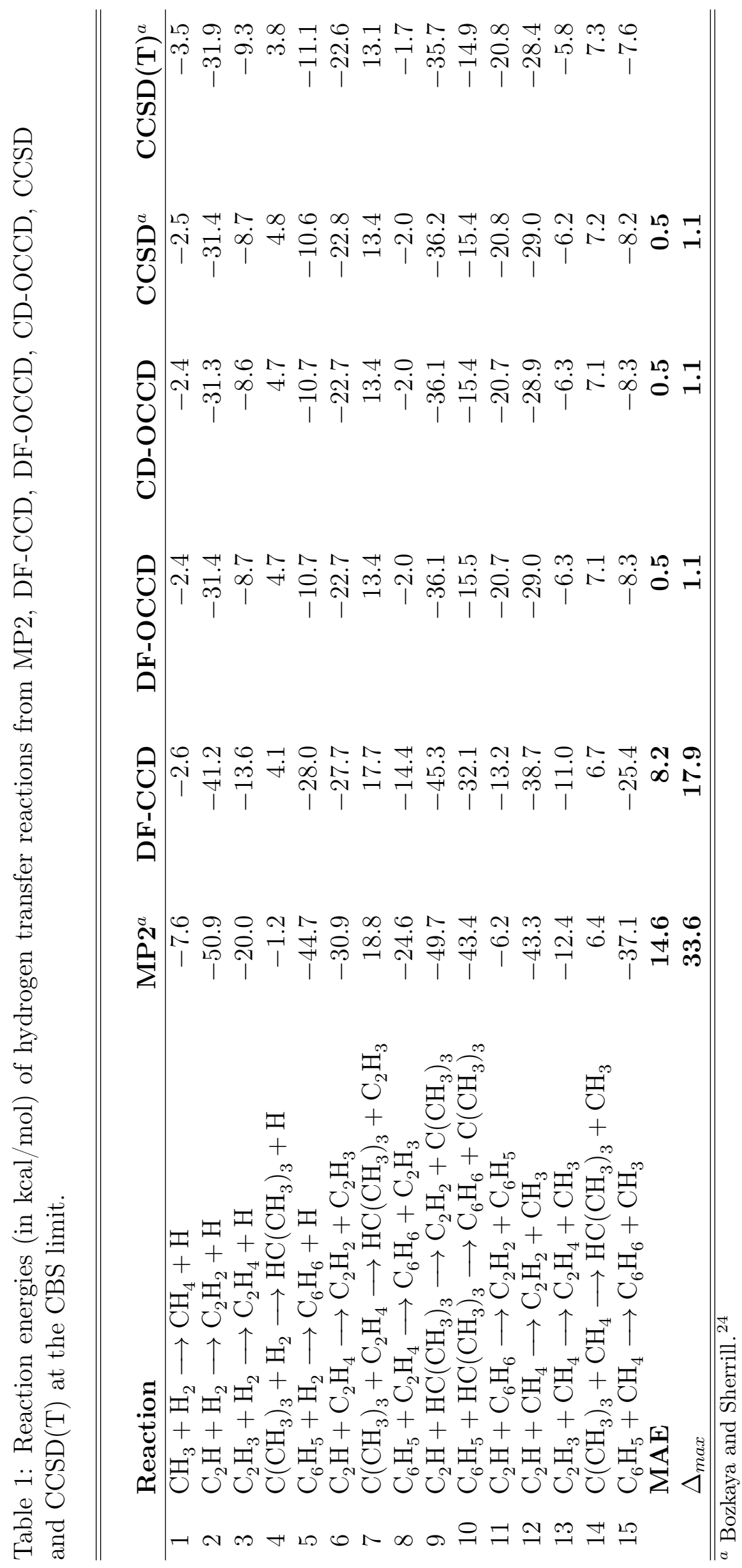




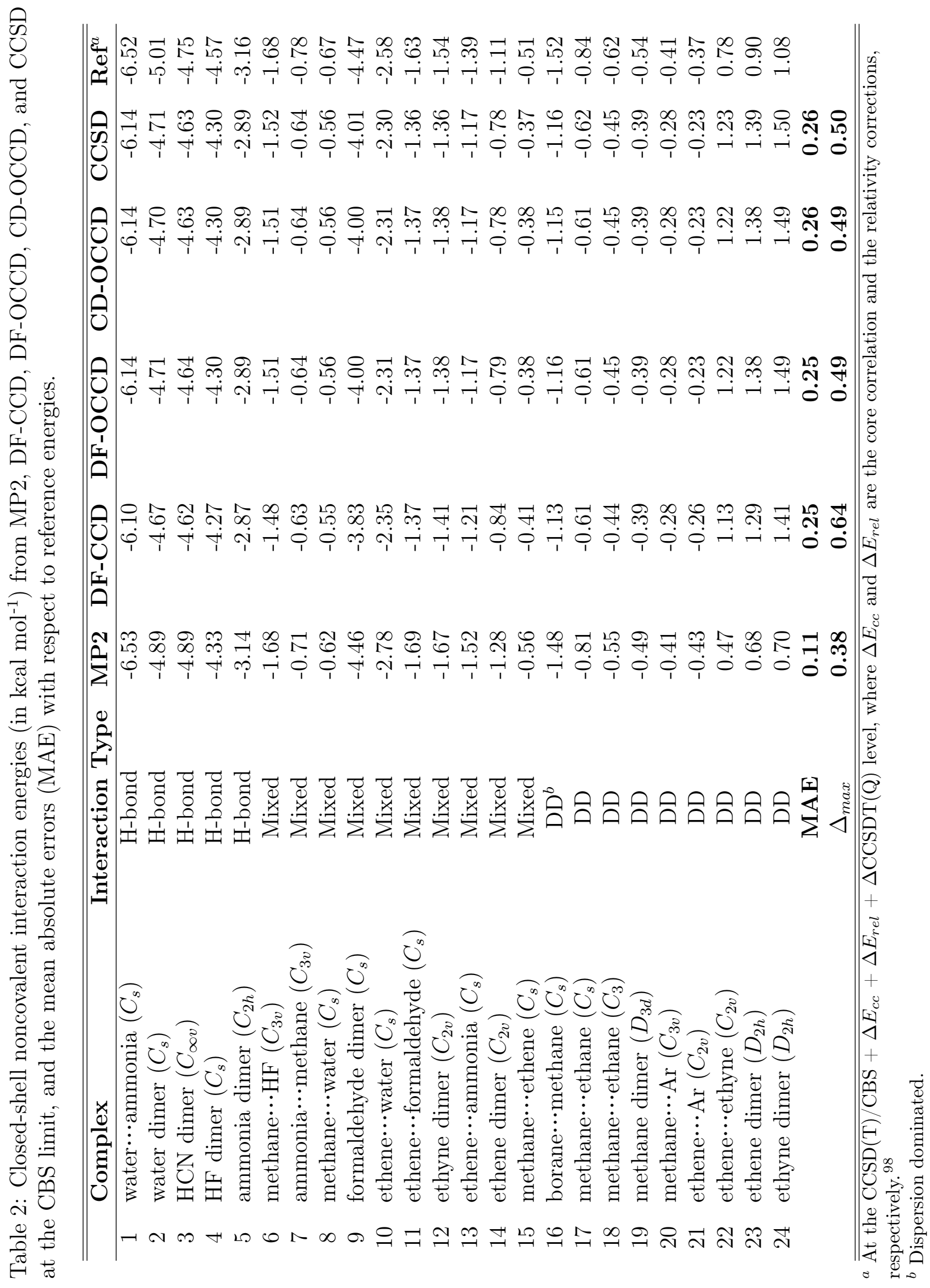


Table 3: Open-shell noncovalent interaction energies (in kcal mol-1) from MP2, DF-CCD, DF-OCCD, CD-OCCD, CCSD, and CCSD $(T)$ at the CBS limit, and the mean absolute errors (MAE) with respect to $\operatorname{CCSD}(\mathrm{T})$.

\begin{tabular}{|c|c|c|c|c|c|c|c|}
\hline & Complex & $\mathrm{MP2}^{a}$ & DF-CCD & "DF-OCCD & "CD-OCCD & $\mathrm{CCSD}^{a}$ & $\overline{C C S D}(\mathrm{~T})^{a}$ \\
\hline 1 & $\mathrm{H}_{2} \mathrm{O} \cdots \mathrm{NH}_{3}^{+}$ & -17.40 & -16.94 & -17.55 & -17.55 & -17.68 & -18.40 \\
\hline 2 & $\mathrm{HOH} \cdots \mathrm{CH}_{3}$ & -1.67 & -1.43 & -1.48 & -1.48 & -1.49 & -1.75 \\
\hline 3 & $\mathrm{NH} \cdots \mathrm{NH}^{b}$ & -1.04 & -1.02 & -1.01 & -1.01 & -1.01 & -1.02 \\
\hline 4 & $\mathrm{Li} \cdots \mathrm{Li}^{c}$ & 0.04 & -0.87 & -0.93 & -0.94 & -0.94 & -0.97 \\
\hline 5 & $\mathrm{H}_{2} \mathrm{O} \cdots \mathrm{HNH}_{2}{ }^{+}$ & -25.58 & -25.16 & -25.11 & -25.10 & -25.09 & -25.41 \\
\hline 6 & $\mathrm{H}_{2} \cdots \mathrm{Li}$ & -0.02 & -0.02 & -0.02 & 0.04 & -0.02 & -0.02 \\
\hline 7 & $\mathrm{FH} \cdots \mathrm{BH}_{2}$ & -4.11 & -3.88 & -3.94 & -3.94 & -3.95 & -4.22 \\
\hline 8 & $\mathrm{He} \cdot \cdots \mathrm{Li}$ & 0.00 & 0.00 & 0.00 & 0.00 & 0.00 & 0.00 \\
\hline 9 & $\mathrm{H}_{2} \mathrm{O} \cdots \mathrm{Al}$ & -7.12 & -6.37 & -6.89 & -6.96 & -6.84 & -7.75 \\
\hline 10 & $\mathrm{Ar} \cdots \mathrm{OH}$ & -0.16 & -0.14 & -0.14 & -0.14 & -0.14 & -0.16 \\
\hline 11 & $\mathrm{FH} \cdots \mathrm{OH}$ & -6.02 & -5.80 & -5.83 & -5.90 & -5.84 & -6.10 \\
\hline 12 & $\mathrm{He} \cdot \mathrm{OH}$ & -0.02 & -0.09 & -0.11 & 0.46 & -0.03 & -0.05 \\
\hline 13 & $\mathrm{H}_{2} \mathrm{O} \cdots \mathrm{Be}^{+}$ & -63.95 & -65.42 & -65.45 & -65.45 & -65.42 & -65.22 \\
\hline 14 & $\mathrm{HF} \cdots \mathrm{CO}^{+}$ & -35.82 & -32.40 & -28.90 & -28.89 & -28.82 & -30.37 \\
\hline 15 & $\mathrm{H}_{2} \mathrm{O} \cdots \mathrm{Cl}$ & -2.95 & -2.10 & -2.57 & -2.57 & -2.66 & -3.58 \\
\hline 16 & $\mathrm{H}_{2} \mathrm{O} \cdots \mathrm{Br}$ & -3.11 & -2.24 & -2.57 & -2.49 & -2.64 & -3.48 \\
\hline 17 & $\mathrm{H}_{2} \mathrm{O} \cdots \mathrm{Li}$ & -11.64 & -12.10 & -12.45 & -12.46 & -12.46 & -12.63 \\
\hline 18 & $\mathrm{FH} \cdots \mathrm{NH}_{2}$ & -10.43 & -9.98 & -10.00 & -10.00 & -10.00 & -10.33 \\
\hline 19 & $\mathrm{NC} \cdots \mathrm{Ne}$ & -0.06 & -0.04 & -0.06 & -0.06 & -0.06 & -0.07 \\
\hline 20 & $\mathrm{He} \cdot \cdot \mathrm{NH}^{c}$ & -0.02 & -0.03 & -0.03 & -0.03 & -0.03 & -0.04 \\
\hline & MAE & 0.60 & 0.50 & 0.36 & 0.38 & 0.34 & \\
\hline & $\Delta_{\max }$ & 5.45 & 2.03 & 1.48 & 1.48 & 1.55 & \\
\hline
\end{tabular}

$\overline{{ }^{a}}$ From Soydaş and Bozkaya. ${ }^{39}$ All systems are in doublet states unless otherwise noted.

${ }^{b}$ The lowest quintet state of the dimer is considered, the lowest singlet and triplet states require multireference wave functions. ${ }^{107}$

${ }^{c}$ The lowest triplet state of the dimer is considered. 


\section{TOC Graphic}
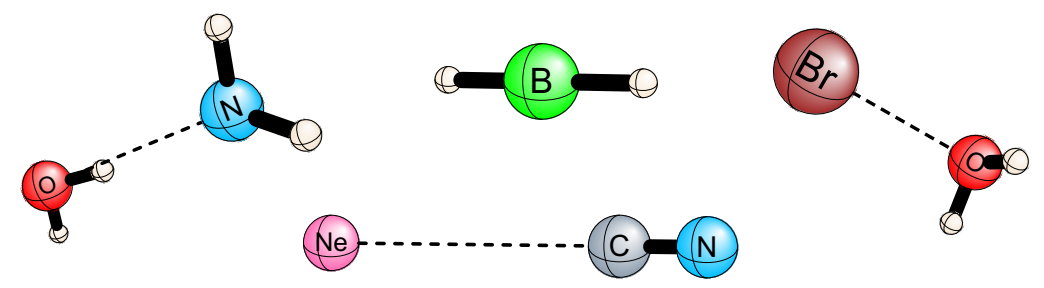

$$
\mathcal{L}(\boldsymbol{\kappa})=\left\langle 0\left|\left(1+\hat{\Lambda}_{2}\right) e^{-\hat{T}_{2}} \hat{H}^{\kappa} e^{\hat{T}_{2}}\right| 0\right\rangle
$$
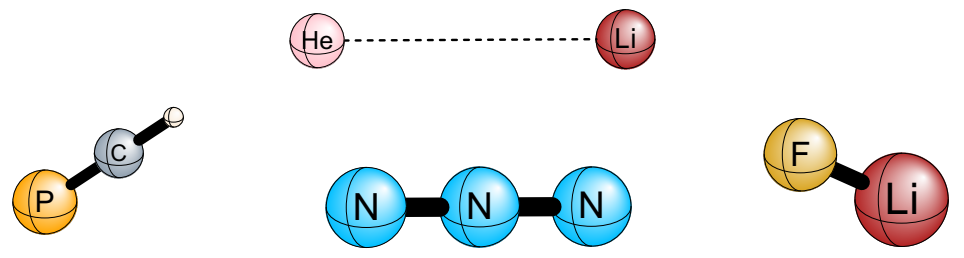